\title{
ROS play a critical role in the differentiation of alternatively activated macrophages and the occurrence of tumor-associated macrophages
}

\author{
Yan Zhang ${ }^{1}$, Swati Choksi ${ }^{1}$, Kun Chen ${ }^{1,2}$, Yelena Pobezinskaya ${ }^{1}$, Ilona Linnoila ${ }^{1}$, Zheng-Gang Liu ${ }^{1}$ \\ ${ }^{1}$ Cell and Cancer Biology Branch, Center for Cancer Research, National Cancer Institute, National Institutes of Health, 37 Con- \\ vent Drive, RM1130, Bethesda, MD 20892, USA; ${ }^{2}$ School of Life Sciences, Nanjing University, Nanjing, Jiangsu 210093, China
}

Differentiation to different types of macrophages determines their distinct functions. Tumor-associated macrophages (TAMs) promote tumorigenesis owing to their proangiogenic and immune-suppressive functions similar to those of alternatively activated (M2) macrophages. We report that reactive oxygen species (ROS) production is critical for macrophage differentiation and that inhibition of superoxide $\left(\mathrm{O}^{2-}\right)$ production specifically blocks the differentiation of $\mathrm{M} 2$ macrophages. We found that when monocytes are triggered to differentiate, $\mathrm{O}^{2-}$ is generated and is needed for the biphasic ERK activation, which is critical for macrophage differentiation. We demonstrated that ROS elimination by butylated hydroxyanisole (BHA) and other ROS inhibitors blocks macrophage differentiation. However, the inhibitory effect of ROS elimination on macrophage differentiation is overcome when cells are polarized to classically activated (M1), but not M2, macrophages. More importantly, the continuous administration of the ROS inhibitor BHA efficiently blocked the occurrence of TAMs and markedly suppressed tumorigenesis in mouse cancer models. Targeting TAMs by blocking ROS can be a potentially effective method for cancer treatment.

Keywords: ROS; alternatively activated M2 macrophages; macrophage differentiation; inflammation; tumorigenesis Cell Research (2013) 23:898-914. doi:10.1038/cr.2013.75; published online 11 June 2013

\section{Introduction}

Macrophages regulate innate immune responses to acute and chronic inflammation [1]. Deregulation of macrophage differentiation may lead to diseases including autoimmune disorders and cancer [2,3]. Circulating monocytes are versatile precursors with the ability to differentiate into various forms of macrophages [4]. Stimulated monocytes activate pro-survival pathways, migrate to tissues and differentiate into macrophages [4]. Granulocyte-macrophage colony-stimulating factor (GM-CSF) and macrophage colony-stimulating factor (M-CSF) induce monocyte-macrophage lineage differentiation both in vivo and in vitro. Previous studies have determined

Correspondence: Zheng-Gang Liu

Tel: +1-301-435-6351; Fax: +1-301-402-1997

E-mail: zgliu@helix.nih.gov

Received 11 March 2013; revised 18 April 2013; accepted 26 April 2013; published online 11 June 2013 many of the signaling pathways activated by GM-CSF or M-CSF $[5,6]$. The three mitogen-activated protein kinases (MAPKs), ERK, JNK and p38, are known to be activated following GM-CSF or M-CSF treatment and have been implicated in monocyte-macrophage differentiation [7-9]. Particularly, an immediate and a late-phase activation of ERK were observed during monocytic cell line differentiation $[7,10]$. Our previous study showed that JNK activation by GM-CSF or M-CSF is critical for monocyte survival and differentiation through mediating the induction of autophagy [11].

GM-CSF- or M-CSF-differentiated macrophages can be further differentiated or polarized to more specialized cells in response to additional stimuli $[12,13]$. For instance, when GM-CSF-differentiated human macrophages are engaged with bacterial products and Th1 cytokines such as lipopolysaccharide (LPS) and interferon- $\gamma$ (IFN $\gamma$ ), they are polarized to classically activated macrophages, also known as M1 macrophages, which harbor immunestimulatory properties and cytotoxic function against tumor cells [14]. However, when M-CSF-differentiated hu- 
man macrophages are activated by Th2 cytokines, such as IL-4, or immune-suppressors, such as IL-10, they become alternatively activated, or M2, macrophages, which have low cytotoxic function but high tissue-remodeling activity $[1,14]$. The molecular mechanism that regulates the differentiation of M1 and M2 macrophages remains enigmatic.

Macrophages are the most abundant immune cells involved in tumor development [15]. Tumor-associated macrophages (TAMs) are M2-like cells and are responsible for many tumor-promoting activities during tumor initiation, progression and metastasis $[16,17]$. TAMs play a major role in suppressing the antitumor responses of dendritic cells (DCs), cytotoxic T lymphocytes (CTLs) and natural killer (NK) cells $[18,19]$. Blocking the functions of TAMs inhibits tumorigenesis [20,21].

ROS are generally considered to be deleterious to cells, but ROS also have an important role in regulating signal transduction pathways [22, 23] and gene expression [24]. Some reports suggested that ROS may be involved in the differentiation of haematopoietic lineages or macrophage cell lines [25], yet little is known about the role of superoxide in the activation of MAPKs during monocyte-macrophage differentiation and in the differentiation of M1 and M2 macrophages.

In this study, we report that ROS play a critical role in the differentiation of M2 macrophages and the occurrence of TAMs. We found that $\mathrm{O}^{2-}$ generation is needed for the late-phase ERK activation and monocytemacrophage differentiation. Particularly, the inhibition of ROS generation specifically affects the polarization to M2, but not M1, macrophages. More importantly, we found that the continuous administration of BHA efficiently blocked the occurrence of TAMs and markedly suppressed tumorigenesis in three different mouse cancer models.

\section{Results}

BHA blocks the differentiation of $M 2$, but not M1, macrophages

To investigate whether necrosis is involved in monocyte death, we tested the effect of BHA, which inhibits necrosis in some cells [26], on the death of human primary monocytes. While BHA had some inhibitory effect on the death of the non-treated cells, surprisingly, we found that the presence of BHA resulted in the loss of GM-CSF- or M-CSF-induced macrophage morphologies of adherent cells and led to most cells being rounded and floating after 6 days in culture (Figure 1A, panels 1, 2 and 4). FACS analysis showed that BHA blocked the increased expression of the macrophage marker, CD11b
(Supplementary information, Figure S1A), suggesting that BHA may affect the monocyte-macrophage differentiation. We then examined whether BHA affects the differentiation of M1 and M2 macrophages. As shown in Figure 1A, panels 3 and 5, when BHA was added before GM-CSF or M-CSF treatment, BHA affected the M2 but not M1 differentiation, as indicated by cell morphologies. However, when BHA was added 6 days after GMCSF or M-CSF treatment, but before the polarization by LPS/IFN $\gamma$ or IL-4, it had no effect on monocyte differentiation to either M1 or M2 macrophages (Supplementary information, Figure S1B). We also found that BHA does not block monocyte-macrophage differentiation when added 1 day after GM-CSF or M-CSF treatment (unpublished data). These data indicated that BHA interferes with macrophage differentiation at the early stage of the process and the inhibitory effect of BHA on GMCSF-differentiated macrophages is overcome during M1 polarization. To confirm that BHA specifically blocked the differentiation of monocytes to M2, but not M1 macrophages, we examined the expression of M1 and M2 macrophage-specific markers. BHA had no effect on the increase of M1 macrophage marker CD86, while it blocked the expression of M2 macrophage marker CD163 [14, 27] (Figure 1B and 1C).

We next investigated the effect of BHA on the production of M1- and M2-specific cytokines and chemokines. BHA had little effect on the production of M1 macrophage cytokines, TNF $\alpha$, IL-12 and IL- 6 and chemokine, CXCL11, but dramatically blocked the production of M2 macrophage cytokine, IL-10, and chemokines, CCL17, CCL18 and CCL24 [14, 28] (Figure 1D-1E). These results suggested that BHA specifically blocked the differentiation of human monocytes to M2 but not M1 macrophages.

\section{ROS are required for $M 2$ macrophage differentiation}

As BHA blocks ROS generation [26], we investigated whether BHA affected M2 macrophage differentiation through eliminating ROS. We first examined whether $\mathrm{O}^{2-}$ was generated following GM-CSF or M-CSF treatment. As shown in Figure 2A, $\mathrm{O}^{2-}$ was generated quickly and reached maximum levels at $12 \mathrm{~h}$ in GM-CSF- or $\mathrm{M}-\mathrm{CSF}$-treated human primary monocytes (Figure 2A and Supplementary information, Figure S2A). Treatment with BHA efficiently inhibited GM-CSF- or M-CSFinduced $\mathrm{O}^{2-}$ production (Figure $2 \mathrm{~A}$ ). To check whether blocking ROS generation by BHA is responsible for its inhibitory effect on macrophage differentiation, we added $\mathrm{H}_{2} \mathrm{O}_{2}$ to the BHA-treated cells. BHA-mediated loss of macrophage morphologies was partially recovered by the presence of low concentrations of $\mathrm{H}_{2} \mathrm{O}_{2}$ (Figure 
A Untreated GM-CSF GM-CSF + LPS + IFMY M-CSF $\mathrm{M}-\mathrm{CSF}+\mathrm{IL}-4$ (M1)

(M2)

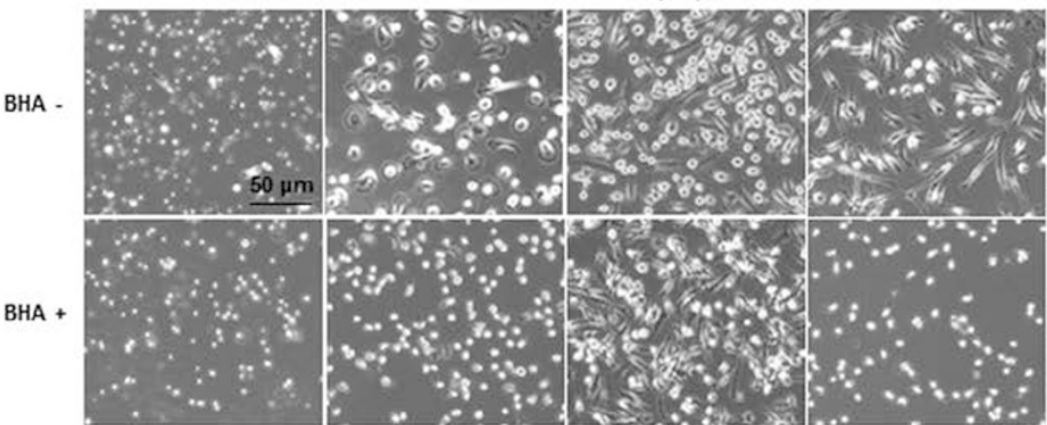

2

4

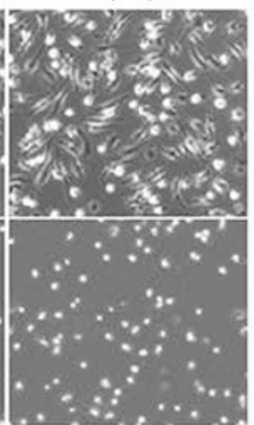

B

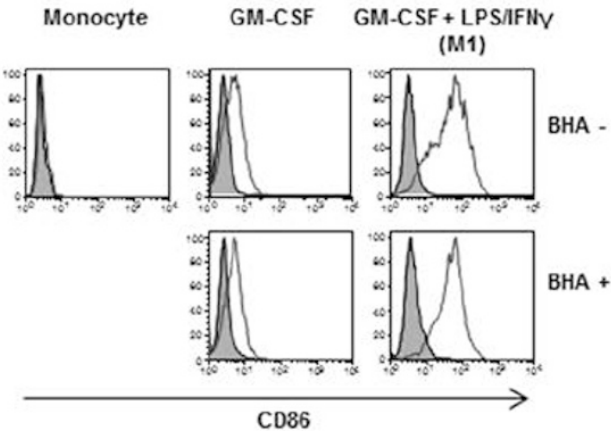

C

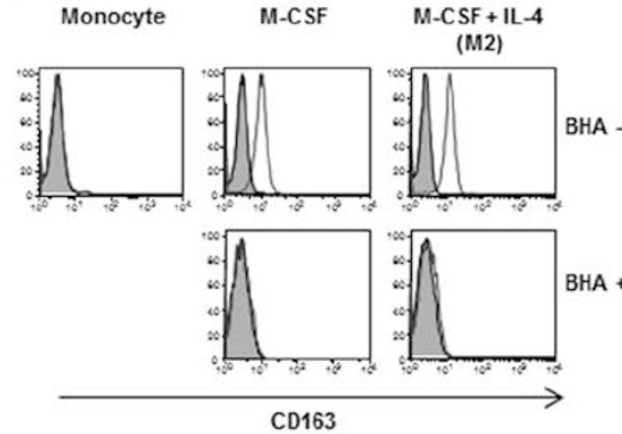

D

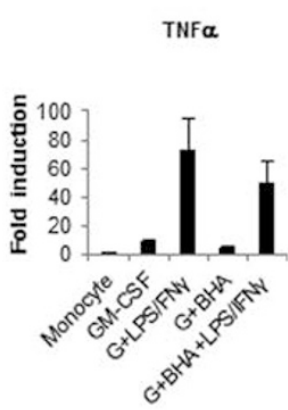

IL-12

E
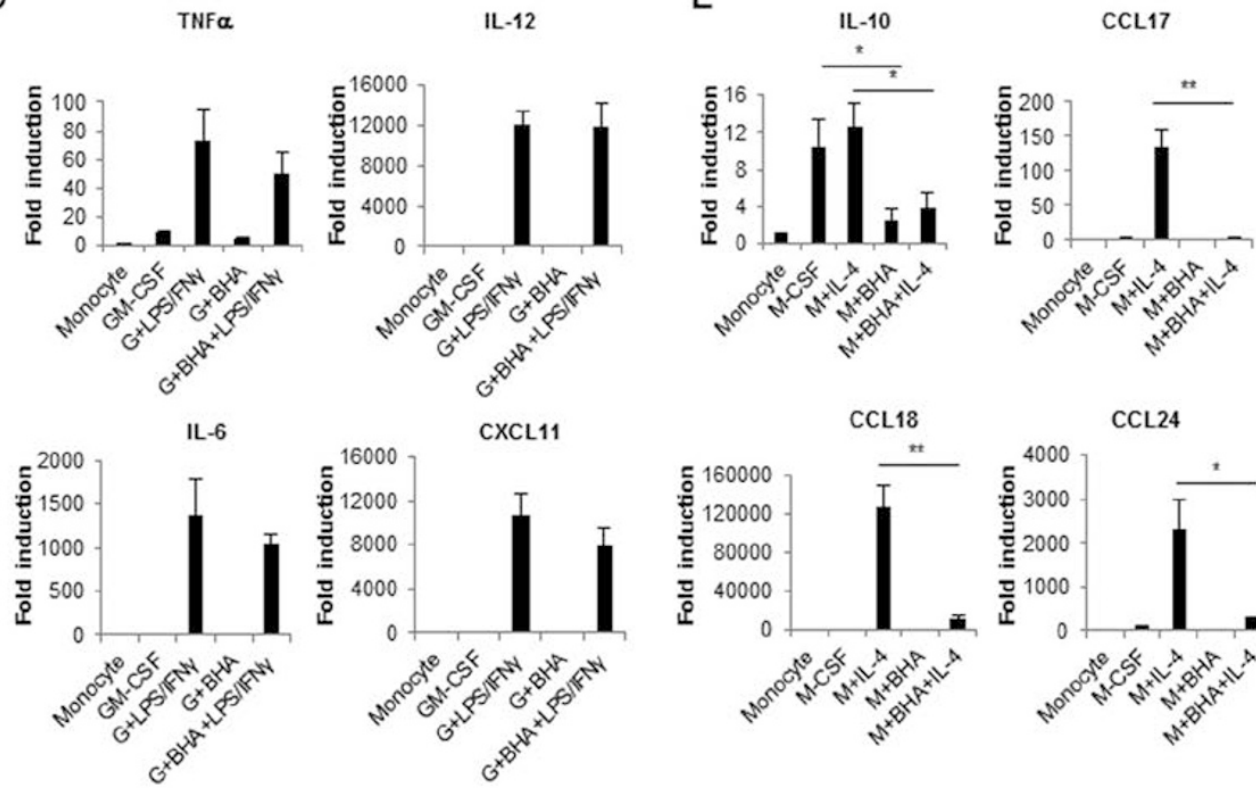

CXCL11
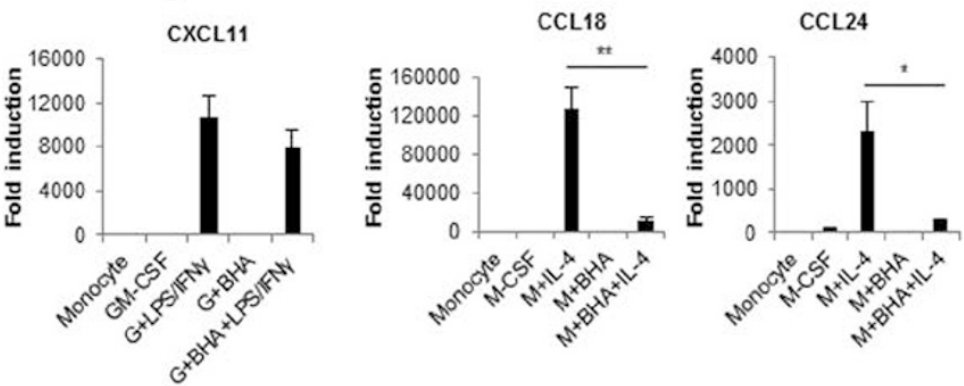

Figure $1 \mathrm{BHA}$ blocks M2 but not M1 macrophage differentiation. (A) Monocytes were either left untreated or treated with BHA $(100 \mu \mathrm{M})$ for $1 \mathrm{~h}$ before GM-CSF or M-CSF treatment for 6 days. On day 6, GM-CSF-treated cells were treated with LPS $(100 \mathrm{ng} / \mathrm{ml})$ and IFN $\gamma(20 \mathrm{ng} / \mathrm{ml})$ for $24 \mathrm{~h}$. M-CSF-treated cells were treated with IL-4 $(25 \mathrm{ng} / \mathrm{ml})$ for $24 \mathrm{~h}$. Representative light microscopy images are shown. (B, C) Flow cytometry analysis of the M1 marker CD86 (B) and M2 marker CD163 (C) with anti-CD86 and anti-CD163 antibodies, respectively, in cells as treated in A. Gray histogram represents unstained cells. (D, E) Detection of M1 cytokines (TNF $\alpha$, IL-12, IL-6) and chemokine (CXCL11) (D), and M2 cytokine (IL-10) and chemokines (CCL17, CCL18, CCL24) (E) by real-time PCR in cells as treated in A. G, GM-CSF; M, M-CSF. Error Bars: \pm SEM, data from at least three independent experiments. ${ }^{*} P<0.05 ;{ }^{* *} P<0.01$. 
A

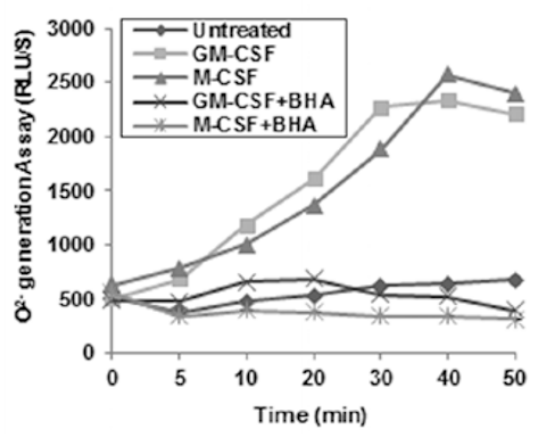

B

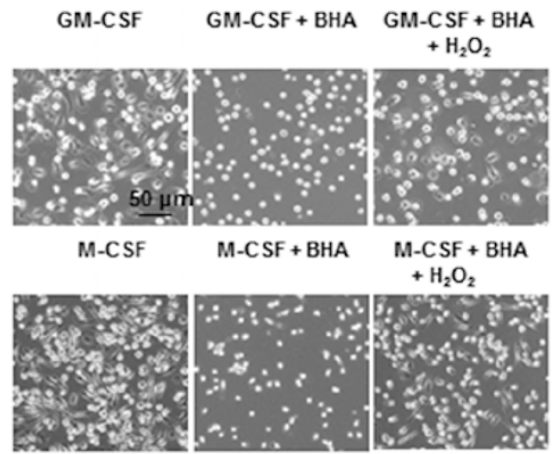

C

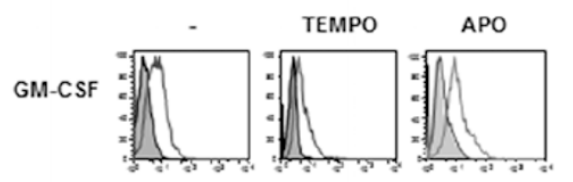

D
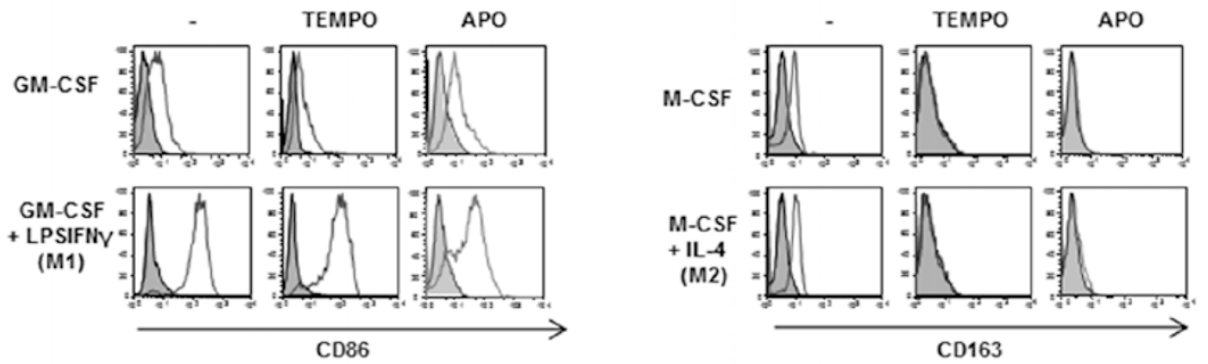

E
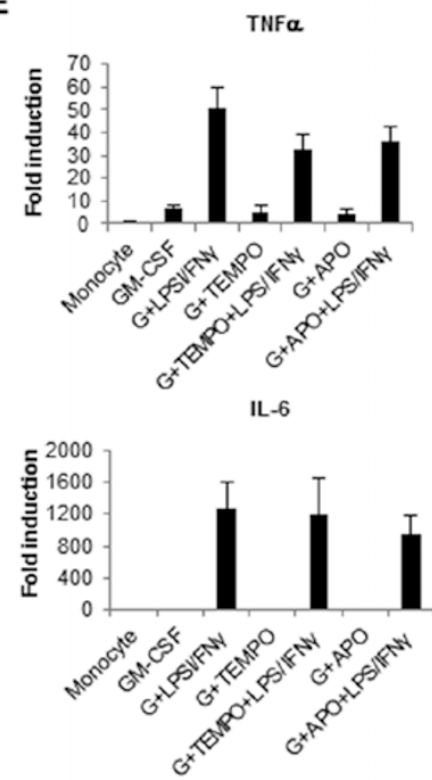

F

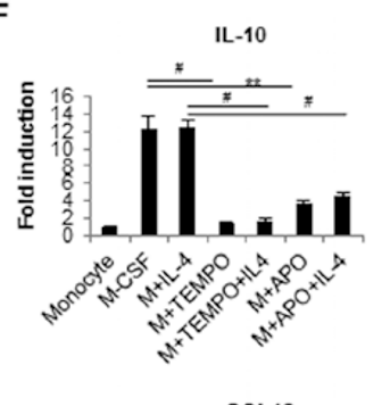

CCL18

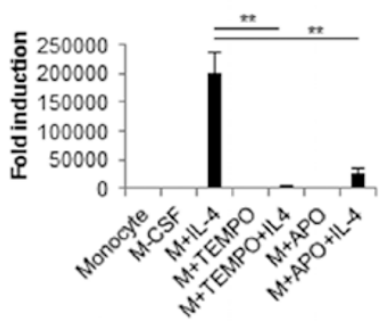

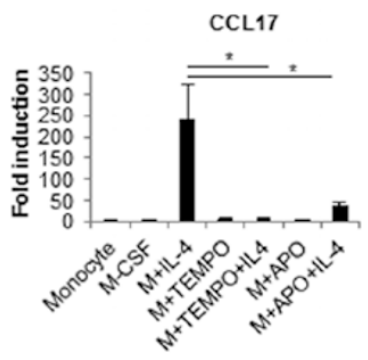

CCL24

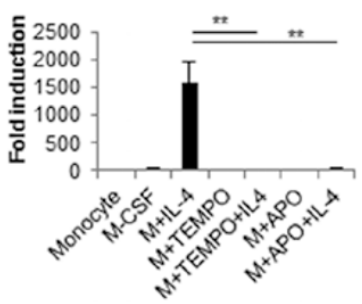

Figure $2 \mathrm{BHA}$ blocks M2 differentiation by inhibiting $\mathrm{O}^{2-}$ generation. (A) Monocytes were either left untreated or pretreated with BHA for $1 \mathrm{~h}$. Cells were then treated with GM-CSF or M-CSF and $\mathrm{O}^{2-}$ generation was measured at the indicated times. (B) Monocytes were either left untreated or pretreated with BHA for $1 \mathrm{~h}$ and then differentiated for 6 days with GM-CSF or MCSF, with or without $\mathrm{H}_{2} \mathrm{O}_{2}(0.001 \mathrm{mM})$. Representative light microscopy images of cells differentiated with GM-CSF or M-CSF are shown. (C, D) Monocytes were either left untreated or treated with apocynin $(500 \mu \mathrm{M})$ or TEMPO $(500 \mu \mathrm{M})$ for $1 \mathrm{~h}$ then differentiated for 6 days with GM-CSF or M-CSF. On day 6, GM-CSF-treated cells were treated with LPS and IFN $\gamma$ (M1) for $24 \mathrm{~h}$. M-CSF-treated cells were treated with IL-4 (M2) for $24 \mathrm{~h}$. Flow analysis of M1 marker CD86 in GM-CSF-treated or polarized M1 cells (C) and M2 marker CD163 in M-CSF-treated or polarized M2 cells (D) are shown. Gray histogram represents unstained cells. (E, F) Detection of M1 cytokines (TNF $\alpha$, IL-6) (E) and M2 cytokines (IL-10) and chemokines (CCL17, CCL18, CCL24) (F) by real-time PCR in M1 and M2 macrophages left untreated or pretreated with TEMPO or apocynin as compared to monocytes. G, GM-CSF; M, M-CSF; APO, apocynin. Error Bars: \pm SEM, data from at least three independent experiments. ${ }^{*} P<0.05 ;{ }^{*} P<0.01 ; \# P<0.001$. 
2B), indicating that ROS play a role in macrophage differentiation. To further confirm the involvement of ROS in macrophage differentiation, we examined the effect of other ROS inhibitors such as apocynin, TEMPO and NAC on the differentiation of monocytes to M1 and M2 macrophages. Apocynin, TEMPO and NAC had no effect on M1 marker CD86, but efficiently inhibited the increase of CD163 expression in M2 macrophages (Figure 2C, 2D and Supplementary information, Figure S2B). TEMPO and apocynin had little effect on the induction of M1 cytokines, TNF $\alpha$ and IL-6, but they dramatically inhibited the expression of the M2 cytokine, IL-10 and the chemokines, CCL17, CCL18 and CCL24 (Figure 2E and $2 \mathrm{~F}$ ). These results indicate that ROS play a key role in the differentiation of $\mathrm{M} 2$ macrophages.

NADPH oxidase (NOX)-mediated superoxide production is the main non-mitochondrial source of ROS. Some reports have described the relevance of NOX-mediated superoxide production in the differentiation of cell types $[25,29]$. To check the role of NOX-generated superoxide in monocyte-macrophage differentiation and macrophage polarization, we knocked down the expression of the small GTPase RAC1, which is an essential component of both NOX1 and NOX2 enzyme complexes [30], in monocytes. The knockdown of RAC1 had no effect on the differentiation of M1 macrophages, but efficiently blocked M2 macrophage differentiation (Supplementary information, Figure S3), suggesting that BHA blocks the differentiation of M2 macrophages through inhibiting $\mathrm{NOX}$-mediated $\mathrm{O}^{2-}$ generation.

ROS-mediated late phase ERK activation is critical for macrophage differentiation

It is reported that MAP kinases, ERK, JNK and p38, are activated and involved in macrophage differentiation [7-9]. To investigate the mechanism of ROS-mediated macrophage differentiation, we examined which MAPK pathway is required for human primary monocyte differentiation with inhibitors specific for each pathway. The inhibition of ERK or JNK pathways, but not the p38 pathway, blocked GM-CSF- or M-CSF-induced monocyte-macrophage differentiation (Supplementary information, Figure S4A). We then tested whether blocking ROS generation by BHA had any effect on these two pathways. We found that GM-CSF and M-CSF induced a biphasic activation of ERK and that BHA particularly inhibited the late-phase ERK activation, although it also had some effect on the early activation (Figure $3 \mathrm{~A}$ and $3 \mathrm{~B})$. In contrast, BHA had no effect on JNK activation (Supplementary information, Figure S4B). Similar results were obtained with apocynin (Supplementary information, Figure S4C). These data implied that GM-CSF- or M-CSF-induced ROS generation is required for latephase ERK activation, which is critical for monocytemacrophage differentiation.

We next examined the role of ERK activation in the differentiation of monocytes to M1 and M2 macrophages. Adding U0126 at the beginning of the differentiation process had little effect on the increase of M1 marker CD86, but blocked the induction of M2 marker CD163 (Figure 3C and 3D). Adding U0126 at this stage did not affect the production of M1 cytokines, TNF $\alpha$ and IL-6, but inhibited the expression of M2 chemokines, CCL17, CCL18 and CCL24 (Supplementary information, Figure S4D). Interestingly, U0126 had no effect on the production of IL-10 in M2 macrophages (Supplementary information, Figure S4D), indicating that besides the ERK pathway, BHA affected additional pathway(s) that is involved in macrophage differentiation. These data indicated that blocking ERK activation in the early stage of macrophage differentiation specifically affects the M2 differentiation.

As BHA and U0126 clearly affected GM-CSF-induced monocyte-macrophage differentiation (Figure 1A and Supplementary information, Figure S4A), it is important to investigate how this defect is overcome during the polarization to M1 macrophages. As LPS activates the ERK pathway through TLR4 in macrophages [27], we then examined ERK activation during M1 polarization. As shown in Figure 3E, top panel, ERK is promptly activated by LPS/IFN $\gamma$ treatment and BHA did not block this activation. Adding U0126 on day 6 after GM-CSF, but not before GM-CSF treatment (day 0), inhibited ERK activation (Figure 3E, bottom panel). Importantly, adding U0126 just before polarizing with LPS/IFN $\gamma$ blocked the induction of the M1 marker CD86 and the production of the M1 cytokine TNF $\alpha$ in BHA-treated cells (Figure 3F and $3 \mathrm{G})$. These results indicated that BHA-caused defects including the loss of ERK activation in GM-CSFdifferentiated macrophages are overcome during M1 polarization. Consistent with this conclusion, ERK is not activated during IL-4-induced M2 polarization (Supplementary information, Figure S4E).

\section{Blocking ROS generation by BHA inhibits M2 mac- rophage differentiation in vivo}

To confirm the effect of BHA on M2 macrophage differentiation in the murine system, we examined the differentiation of mouse bone marrow cells. M-CSF differentiates bone marrow cells into bone marrow-derived macrophages (BMMs). Similar to human macrophages, murine BMMs treated with IFN $\gamma / \mathrm{LPS}$ polarize to M1 macrophages with high expression of iNOS, a specific marker of murine M1 macrophages [14, 31]. BMMs are 
A

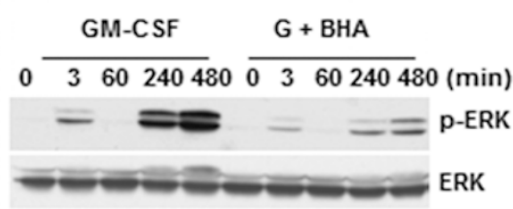

C

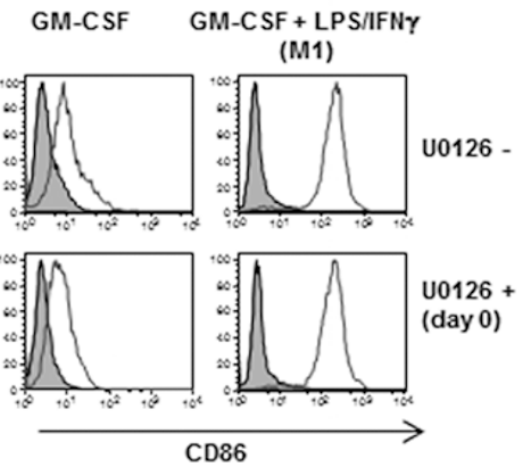

E
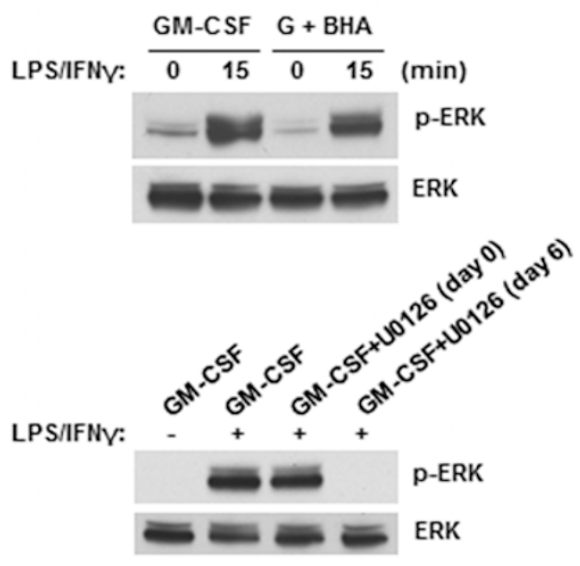

G

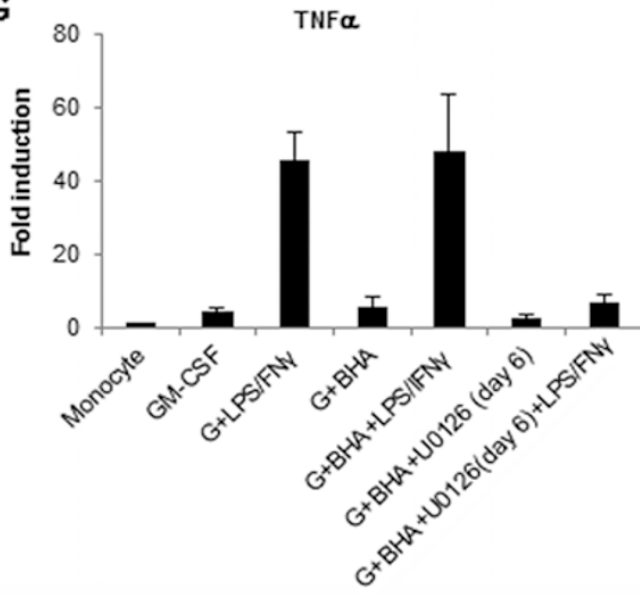

Figure 3 Late-phase ERK activation is critical for M2 macrophage differentiation. (A, B) Monocytes were pretreated with or without BHA for $1 \mathrm{~h}$ and then treated with GM-CSF (A) or MCSF (B) for the indicated times. Cell lysates were immunoblotted with the indicated antibodies. G, GM-CSF; M, M-CSF. (C, D) Monocytes were pretreated or without ERK inhibitor (U0126, $5 \mu \mathrm{M})$ for $1 \mathrm{~h}$. Cells were differentiated for 6 days with GM-CSF or M-CSF. On day 6 , cells were further differentiated by adding
B

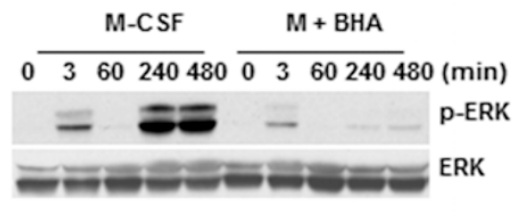

$\mathrm{D}$

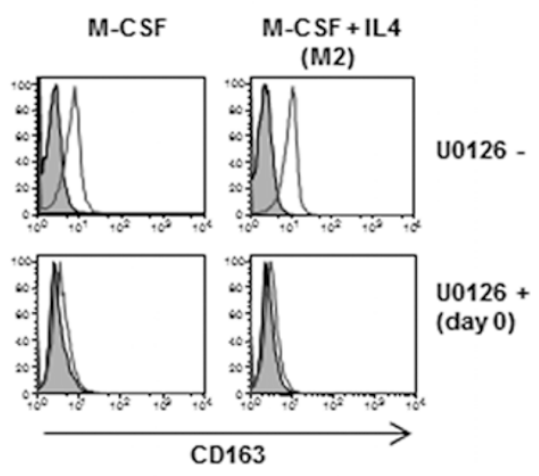

F

GM-CSF GM-CSF + LPS/IFN $\gamma$ (M1)
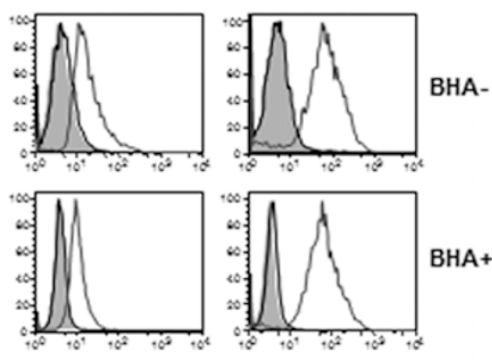

BHA+
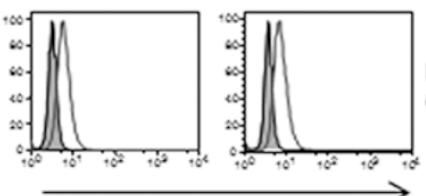

$\mathrm{BHA}+\mathrm{U} 0126$ (day 6)

LPS/IFN $\gamma$ or IL-4 for $24 \mathrm{~h}$. Flow cytometry analysis of the CD86 in GM-CSF-treated or polarized M1 cells (C) and CD163 in MCSF-treated or polarized M2 cells (D) are shown. (E) Upper panel, monocytes were pretreated with or without BHA for $1 \mathrm{~h}$ on day 0 and then treated with GM-CSF for 6 days. On day 6 , cells were treated with LPS/IFN $\gamma$ for the indicated times. Lower panel, monocytes were differentiated with GM-CSF for 6 days. On day 6, cells were treated with LPS/IFN $\gamma$ for $15 \mathrm{~min}$. U0126 was added on either day 0 or day 6 . Cell lysates were immunoblotted with the indicated antibodies. (F) Monocytes were pretreated with or without $\mathrm{BHA}$ for $1 \mathrm{~h}$ and then treated with GM-CSF for 6 days. On day 6 , cells in BHA-treated group were pretreated with or without U0126 for $1 \mathrm{~h}$, and then treated with or without LPS/IFN $\gamma$ for $24 \mathrm{~h}$. Cells were collected and analyzed by FACS with anti-CD86 antibody. Gray histogram represents unstained cells. (G) Monocytes were pretreated with or without $\mathrm{BHA}$ for $1 \mathrm{~h}$ and then treated with GM-CSF for 6 days. On day 6 , BHA-treated cells were then treated with or without U0126 for 1 $\mathrm{h}$ followed by LPS/IFN $\gamma$ for $24 \mathrm{~h}$. Fold change of TNF $\alpha$ detected by real-time PCR compared to monocytes. G, GM-CSF. Error Bars: \pm SEM, data from at least three independent experiments. 
treated with IL-4 or IL-4 plus IL-10 to polarize to M2 macrophages [31, 32], which have specific markers Arginase I, Ym-1 and RELM $\alpha /$ FIZZ1 [14]. BMMs, M1 and M2 macrophages are characterized by the expression of cell surface marker F4/80 [33, 34] and loss of monocyte marker Ly6C. BHA blocked the expression of F4/80 in BMMs and M2, but had a modest effect on F4/80 expression in murine M1 macrophages (Figure 4A). Con- sistently, BHA abolished the Arginase I expression in M2 macrophages and did not affect the iNOS level in M1 macrophages (Figure 4B). In addition, while BHA did not block TNF $\alpha$ production in M1 macrophages, it inhibited IL-10 expression in M2 macrophages (Figure 4C). These data suggest that, similar to the human system, BHA specifically blocked the differentiation of murine monocytes to M2 macrophages.
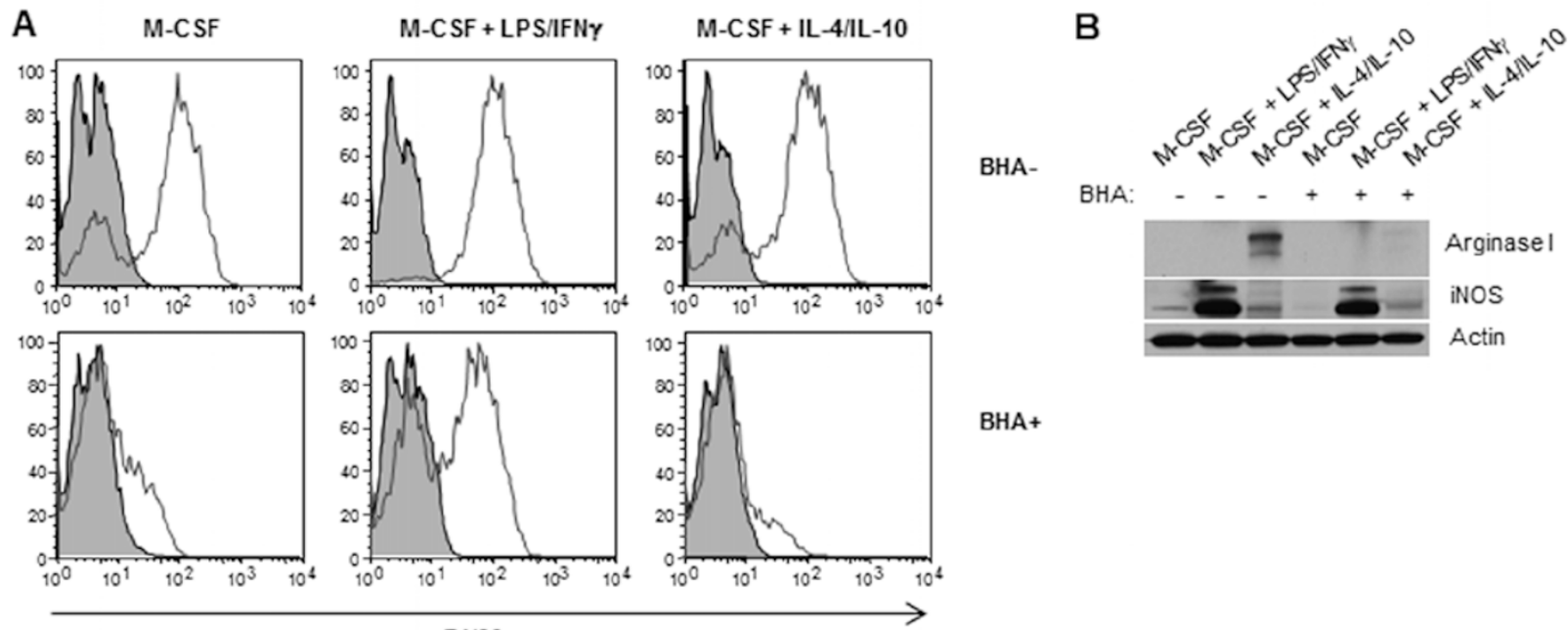

$\mathrm{BHA}+$
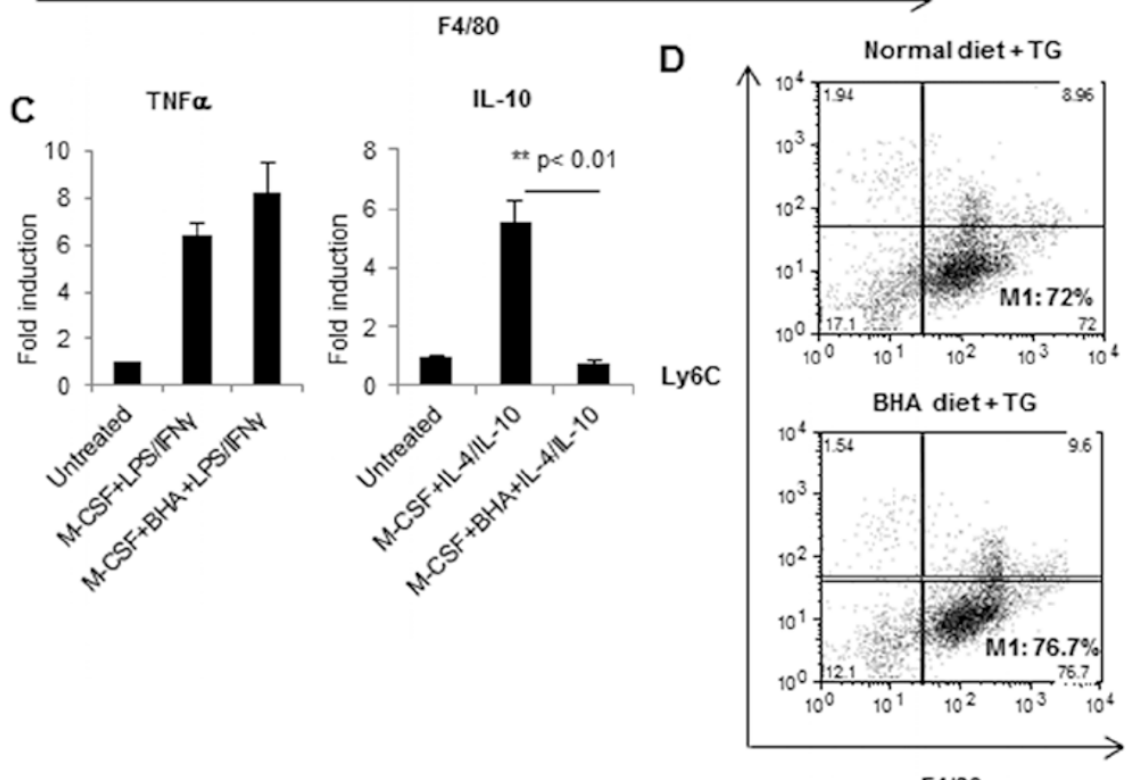

E

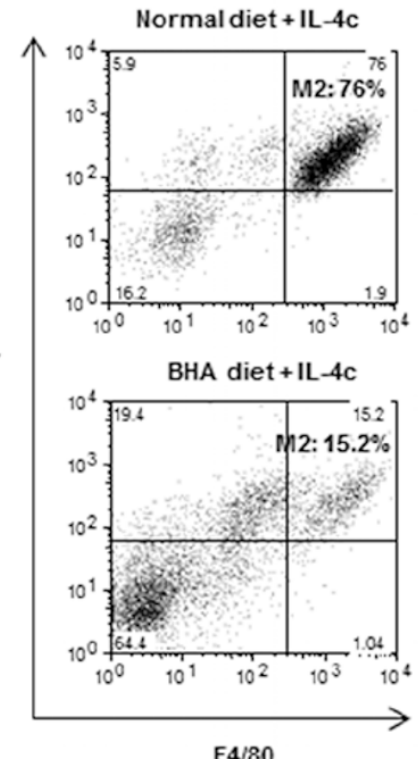

Figure 4 BHA blocks M2 but not M1 polarization in mouse bone marrow-derived macrophages. (A) Mouse bone marrowderived cells were either treated with or without BHA for $1 \mathrm{~h}$ followed by M-CSF for 6 days. On day 6 , cells were either further differentiated with LPS and IFN $\gamma$ or IL-4 and IL-10 for $24 \mathrm{~h}$. Cells were collected, stained with anti-F4/80 antibody and analyzed by flow cytometry. (B) The lysates of the cells from A were also analyzed by western blotting with anti-arginase I, anti-iNOS and anti-actin antibodies. (C) Detection of M1 cytokine (TNF $\alpha$ ) or M2 cytokine (IL-10) by real-time PCR in M1 or M2 macrophages pretreated with or without BHA compared with monocytes. Error Bars: \pm SEM, data from at least three independent experiments. (D, E) Mice were maintained on either normal or BHA diet for two weeks then i.p. injected with either thioglycolate (TG) (D) or IL-4c (E). After 4 days macrophages were isolated by peritoneal lavage. These cells were co-stained with the indicated antibodies and analyzed by flow cytometry. Representative images are shown. 
Thioglycollate stimulates the classical inflammatory cascade and leads to the recruitment of M1 macrophages to the peritoneal cavity, while the combination of IL-4 and anti-IL-4 antibody (referred to as IL-4c) results in the accumulation of RELM $\alpha^{+} \mathrm{M} 2$ macrophages [35]. To examine whether BHA affects the differentiation of M2 macrophages in vivo, mice were maintained continuously on either normal or BHA diet for 2 weeks and then injected with thioglycollate or IL-4c. The formulation of BHA diet was adapted from a previous study on the effect of BHA on tumor development [36]. As shown in Figure 4D, thioglycollate treatment resulted in similar numbers of peritoneal $\mathrm{F} 4 / 80^{+} / \mathrm{Ly} 6 \mathrm{C}^{-} \mathrm{M} 1$ macrophages in mice on normal and BHA diet. In contrast, BHA treatment of IL-4c-treated mice dramatically reduced the peritoneal accumulation of $\mathrm{F} 4 / 80^{+} / \mathrm{RELM}^{+} \mathrm{M} 2 \mathrm{mac}-$ rophages (Figure 4E). These results indicated that BHA efficiently inhibits the differentiation of M2 macrophages in vivo.

\section{BHA blocks the occurrence of TAMs and suppresses tum- origenesis}

As M2 macrophages share many features with TAMs, which are critical mediators of tumorigenesis [16], we then tested whether BHA blocks the occurrence of TAMs in tumor models. Considering that monocytes are constantly produced from bone marrow, we reasoned that continuous BHA treatment is necessary for blocking the differentiation of monocytes to TAMs in three different cancer models. The continuous BHA treatment is not toxic to mice as we did not notice any developmental abnormalities or diseases including cancer in mice on BHA diet for up to 2 years. The first model that we used is the Urethane-induced lung cancer model in which TAMs are thought to play a role in tumorigenesis [37]. $\mathrm{A} / \mathrm{J}$ mice were maintained on BHA diet 2 weeks before or 1 week after Urethane treatment, while control animals were left on normal diet (Supplementary information, Figure S5A). Whole lungs from these mice were collected 6 months after Urethane injection. To quantify the presence of macrophages, lung samples were stained with anti-F4/80 antibody. As shown in Figure 5A, similar background levels of $\mathrm{F} 4 / 80^{+}$macrophages are present in the lungs of control animals on normal and BHA diet. The numbers of $\mathrm{F} 4 / 80^{+}$cells are dramatically increased, especially in the areas around the tumors, in the lungs of Urethane-treated mice compared with non-Urethane control mice. Administration of BHA either before or after Urethane treatment almost completely blocked the increase of macrophages in the lungs (Figure 5A and 5B). To verify that the increased macrophages in Urethanetreated mice are mostly M2-like TAMs, we examined the expression of M2 macrophage markers Arginase I and Ym-1 in these lung samples by western blotting. As shown in Figure 5C and Supplementary information, Figure S5B, very low or no expression of Arginase I and $\mathrm{Ym}-1$ was detected in the lungs of control mice on normal or BHA diet. However, the expression levels of Arginase I and Ym-1 were dramatically increased in the lungs of Urethane-treated mice and importantly, this elevated expression of Arginase I and Ym-1 was abolished when BHA was administered to Urethane-treated mice (Figure 5C and Supplementary information, Figure S5B). These results indicated that BHA blocks the occurrence of TAMs in the Urethane-induced lung cancer model.

We examined the lung tumor multiplicity and tumor burden by microscopic examination after serial sectioning of lungs, as previously described [38, 39]. As shown in Figure 5D-5G and Supplementary information, Figure $\mathrm{S} 5 \mathrm{C}, \mathrm{A} / \mathrm{J}$ mice developed large numbers of lung tumors 6 months after Urethane treatment, and administration of BHA before or after Urethane treatment significantly blocked tumor development, although less effectively when BHA is administered after Urethane treatment. As both tumor multiplicity and tumor burden are reduced by BHA, these results indicated that BHA blocks tumorigenesis at both initiation and progression stages.

Similar studies were performed using two oncogenedriven cancer models: $K$-ras ${ }^{L A 2}$-induced lung cancer and $M M T V-P y M T$-induced breast cancer [40, 41]. Threeweek-old male $K$-ras ${ }^{L A 2}$ mice were weaned and started on normal or continuous BHA diet (Supplementary informa-

Figure 5 BHA blocks the occurrence of TAMs and suppresses tumorigenesis in Urethane-induced tumor model. (A, B) A/ $\mathrm{J}$ mice were maintained on normal or BHA diet for 2 weeks prior to Urethane injection (A) or mice on normal or BHA diet 1 week after Urethane treatment (B). Lungs were collected 6 months after Urethane treatment, sectioned and immunostained for F4/80. Representative image is shown in the left panel ( $T$, tumor area) and quantitative analysis is shown in the right panel. The $\mathrm{F} 4 / 80^{+}$cells were evaluated by counting 10 high power fields $(20 \times)$ per lung section $(1 \mathrm{~mm}) /$ two tissue sections/ mouse. $n=10$ mice. (C) Lung tissues from A were analyzed by western blotting with anti-arginase I, anti-YM-1 and anti-actin antibodies. (D) Representative H\&E images of lungs of mice from A. (E) Lung tumor multiplicity of mice from (A) was determined by counting total tumor foci in 5 serial sections at $400 \mathrm{~mm}$ intervals. (F) Representative H\&E images of lungs of mice from B. (G) Lung tumor multiplicity of mice from (B) was determined by counting total tumor foci in five serial sections at 400 $\mathrm{mm}$ intervals. Error Bars: \pm SEM. 
A

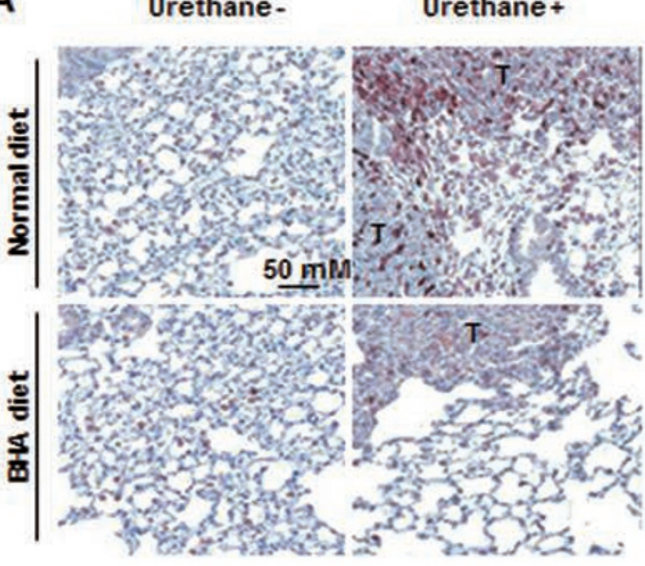

B

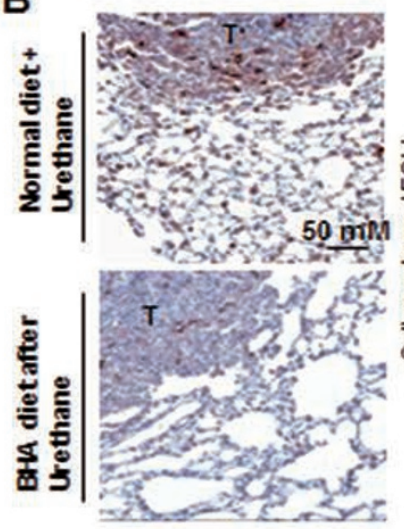

D

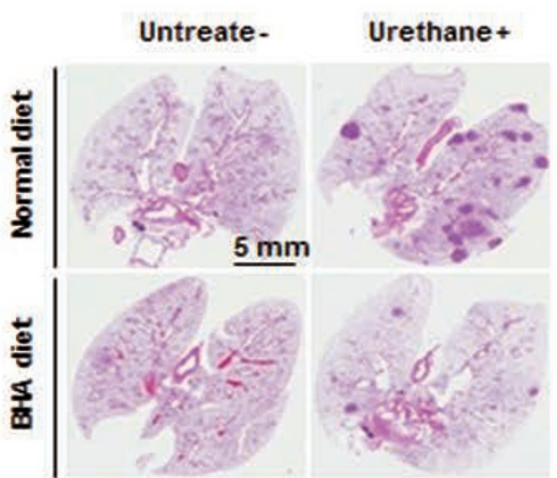

F

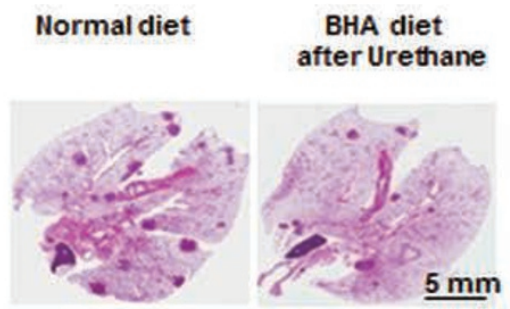

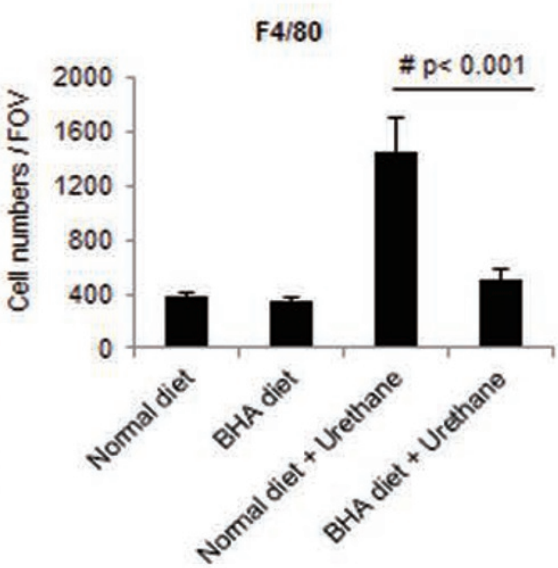

C

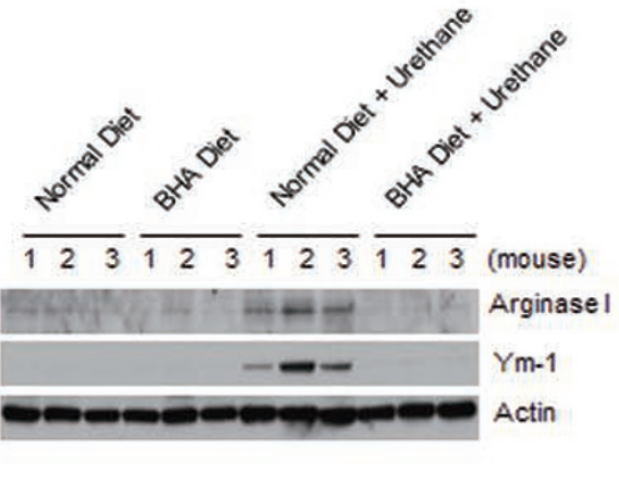

E

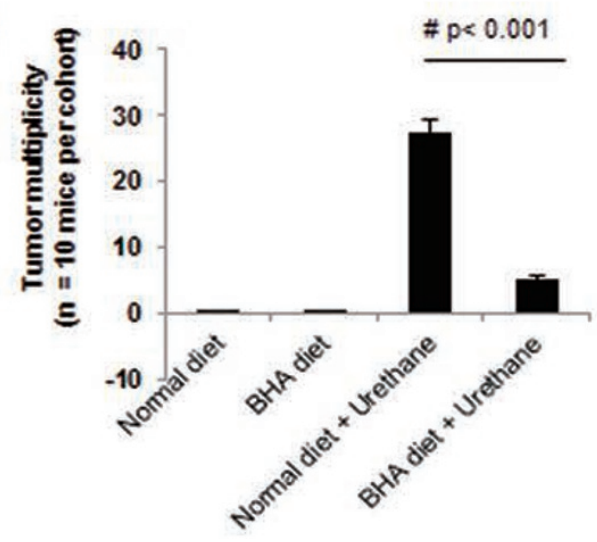

G

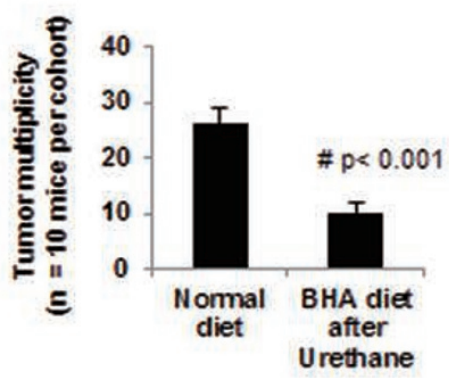


tion, Figure S5A). Whole lungs of these mice were collected at 5 months of age and the effect of BHA on the occurrence of TAMs in $K-$ ras $^{L A 2}$ tumor-bearing mice was analyzed. High levels of $\mathrm{F} 4 / 80^{+}$macrophages were present in the lungs of $K-\mathrm{ras}^{L A 2}$ mice and BHA dramatically reduced the number of $\mathrm{F} 4 / 80^{+}$cells (Figure 6A). The elevated expression of M2 marker Arginase I in $\mathrm{K}$-ras ${ }^{L A 2}$ mice was also abolished by BHA (Figure 6B). Compared with the mice on normal diet, $K-\operatorname{ras}^{L A 2}$ mice on BHA diet had a significant decrease in lung tumor multiplicity and reduction in lung tumor size (Figure 6C-6E and Supplementary information, Figure S6). While most $K$ $\mathrm{ras}^{L A 2}$ mice on normal diet are dead at 6 months of age, the mice on BHA diet have a much longer lifespan (Figure $6 \mathrm{~F})$.

In the $M M T V-P y M T$ breast cancer model, the latestage carcinogenesis and pulmonary metastasis are regulated by TAMs $[42,43]$. To test whether BHA blocks the occurrence of TAMs in this model, 3-week-old, weaned female MMTV-PyMT mice were put on normal or continuous BHA diet and mammary tissues/tumors and whole lungs of these mice were collected at 100 days of age (Supplementary information, Figure S5A). F4/80 macrophages are dramatically reduced in the mammary tissue/tumor samples of mice on BHA diet as compared with samples from mice on normal diet (Figure 7A). Importantly, BHA significantly reduced the mammary tumor burden and numbers of metastatic foci in the lungs of mice on BHA diet compared with those of mice on normal diet (Figure 7B-7D).

These results indicated that BHA inhibited the occurrence of TAMs and suppressed the development of primary tumors in the K-ras ${ }^{L A 2}$ and MMTV-PyMT models and possibly, tumor metastasis in the MMTV-PyMT breast cancer model.

BHA has no effect on MCP-1-directed monocyte migration and tumor cell growth

As monocytes are recruited to the tumor tissue and differentiate into TAMs, we tested whether BHA affected monocyte recruitment. We used MCP-1 (CCL2), which is the major chemokine regulating monocyte recruitment [16], as the chemoattractant in a monocyte transwell migration assay in the absence or presence of BHA. As shown in Figure 8A, MCP-1 significantly increased cellular migration of human monocytes (five- to six-fold increase compared with control group), and the presence of BHA had no effect on MCP-1-induced monocyte migration (about five-fold increase compared with BHA control).

To further prove that BHA suppresses tumorigenesis by blocking M2 macrophage/TAM differentiation, we examined the effect of BHA on tumor cell growth in vitro and in vivo. As shown in Figure $8 \mathrm{~B}$, the presence of BHA had no effect on the proliferation of three types of tumor cell lines. In addition, we tested the effect of BHA on tumor growth in breast cancer xenograft model by inoculating MDA-MB-231-tdTomato cells in athymic $\mathrm{nu} / \mathrm{nu}$ mice. Animals were maintained on normal NIH31 chow or NIH-31 chow with $7.5 \mathrm{~g} / \mathrm{kg}$ BHA starting 1 week before the inoculation of tumor cells. We found that both mice fed with normal food and mice with BHA food formed primary tumors, while the presence of BHA resulted in significant reduction of tumor size 6-8 weeks post-inoculation compared with control group, suggesting that BHA inhibits tumor growth in this model (Figure $8 \mathrm{C})$. To prove that the inhibition of tumor growth by BHA was caused by its effect on the tumor-associated macrophages, but not on tumor cell proliferation, we next studied the effects of macrophage depletion on tumor progression with or without BHA in this model. Macrophage depletion was achieved by treating nude mice with liposomal clodronate, which induced $80 \%$ $90 \%$ depletion of macrophages monitored by testing for the peritoneal macrophages and spleen macrophages (data not shown). As shown in Figure 8C, liposomal clodronate treatment inhibited tumor growth in both normal diet group and BHA diet group. However, the presence of BHA did not induce the suppression of tumor growth after macrophage depletion (Figure 8C). Our data suggest that BHA blocks tumor growth by specifically affecting TAMs but not the proliferation of tumor cells directly.

\section{Discussion}

Macrophages are chief participants in host inflammatory responses. The underlying mechanisms of macrophage differentiation and polarization are still poorly understood. Using human primary monocytes and murine bone marrow-derived cells, we demonstrated the important role of ROS in M2 macrophage differentiation. Our results showed that continuous administration of BHA blocks the occurrence of TAMs and suppresses tumorigenesis.

In macrophages, the generation of superoxide $\left(\mathrm{O}^{2-}\right)$ is important for them to kill invasive microorganisms. However, the role of ROS in macrophage differentiation has not been well defined. Here, we found that ROS is produced in the early stage of monocyte-macrophage differentiation. Blocking ROS generation by BHA, TEMPO, NAC and apocynin specifically inhibits M2 macrophage differentiation. Knocking down RAC1, which is one of the critical ROS-producing components 
A

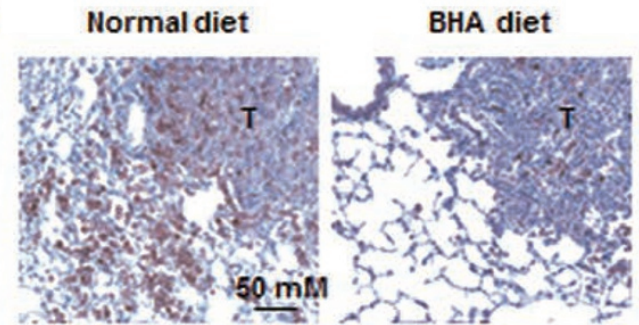

B
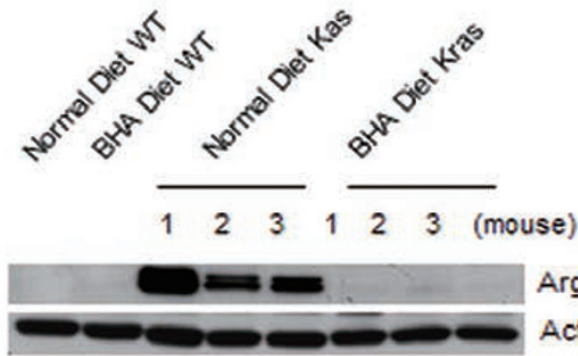

Arginase I Actin

C

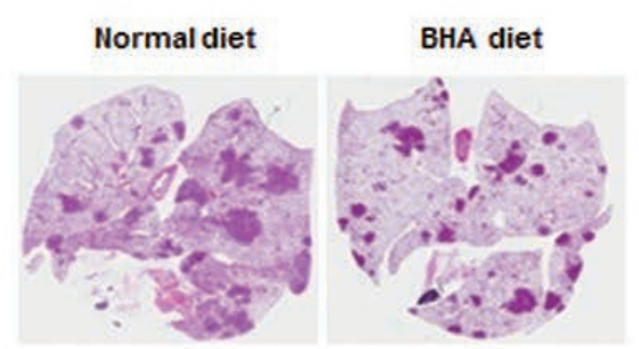

E
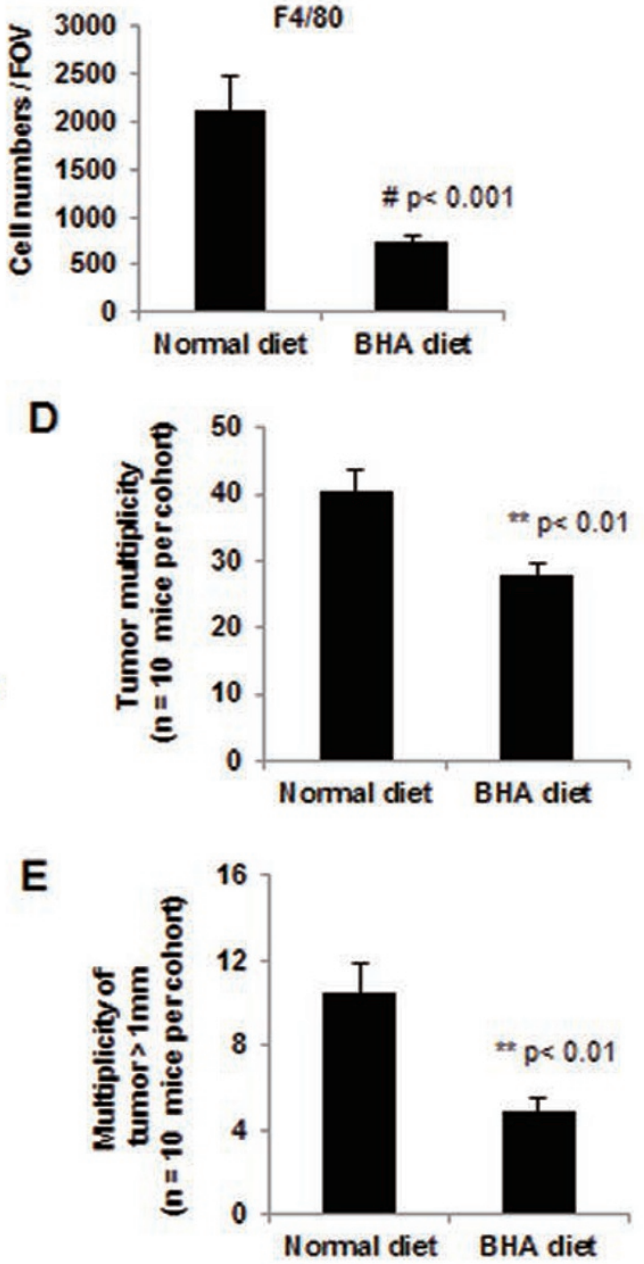

Normal diet BHA diet

$\mathbf{F}$ Kras ${ }^{\mathrm{LA2}}$ Mice Survival Curve

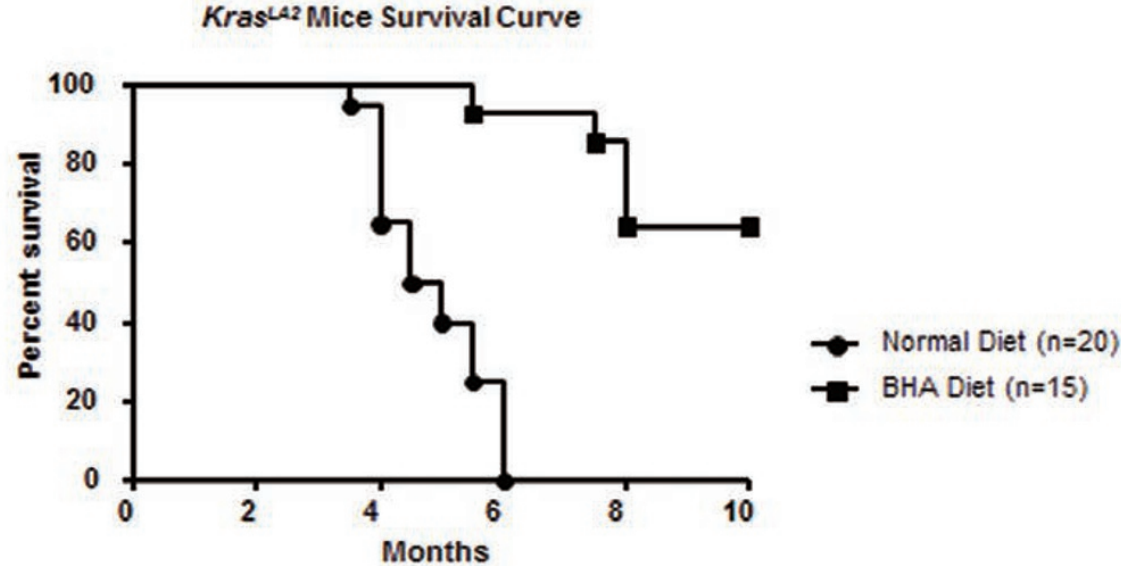

Figure 6 BHA inhibits TAM occurrence and suppresses lung adenomas in $K$-ras ${ }^{L A 2}$ mice. (A) $K$-ras ${ }^{\mathrm{LA2}}$ mice were maintained on normal or BHA diet at 3 weeks of age. Lungs were collected at 5 months of age, sectioned and immunostained for F4/80. Representative image is shown in the left panel ( $T$, tumor area) and quantitative analysis is shown in the right panel. The $\mathrm{F} 4 / 80^{+}$cells were evaluated by counting 10 high power fields $(20 \times)$ per lung section $(1 \mathrm{~mm}) / 2$ tissue sections $/ \mathrm{mouse}$. $n=6$ mice. (B) Lung tissues from A were analyzed by western blotting with anti-arginase I and anti-actin antibodies. (C) Representative H\&E images of lungs of mice from $\mathbf{A}$. (D, E) Lung tumor multiplicity of total lung (D) and large tumors (over $1 \mathrm{~mm}$ in size, E) of mice from $A$ was determined by counting total tumor foci in five serial sections at $400 \mathrm{~mm}$ intervals. Error Bars: \pm SEM. (F) Survival curve of $K$-ras ${ }^{L A 2}$ mice maintained on BHA or normal diet from 3 weeks of age is shown. 
A

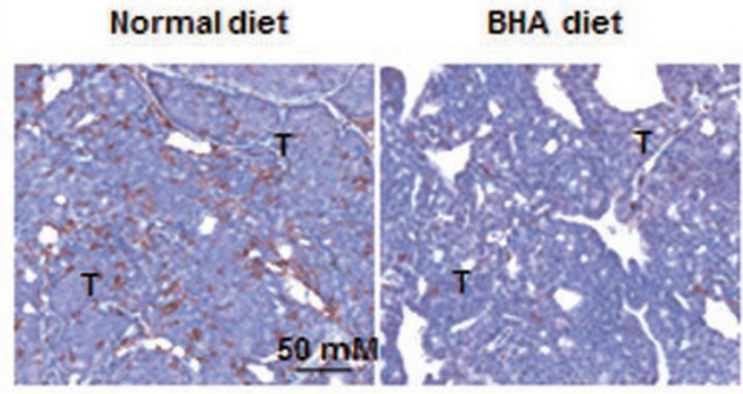

B

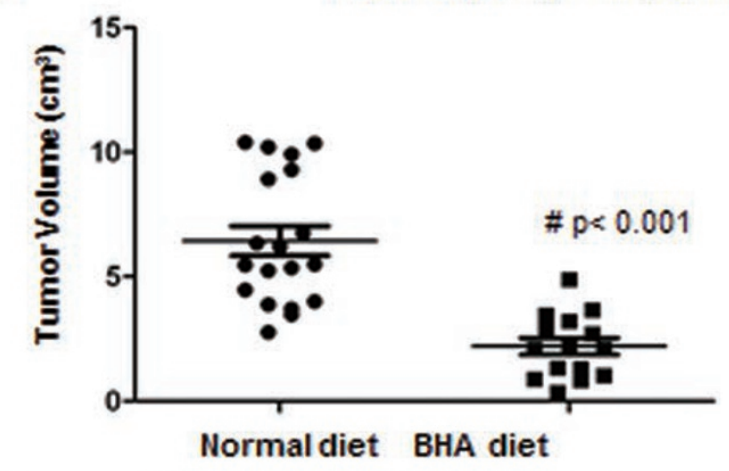

C MMTV-PYMT lung metastasis Normal diet

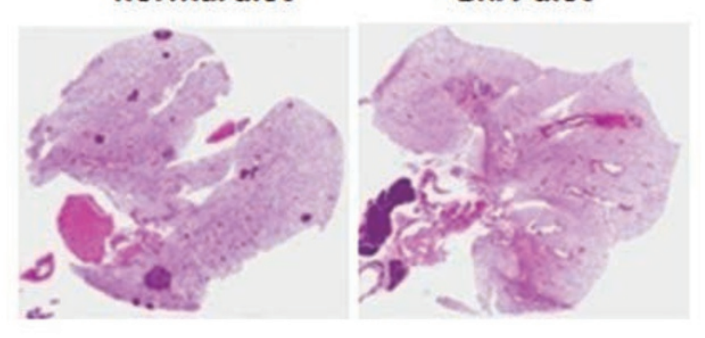

F $4 / 80$

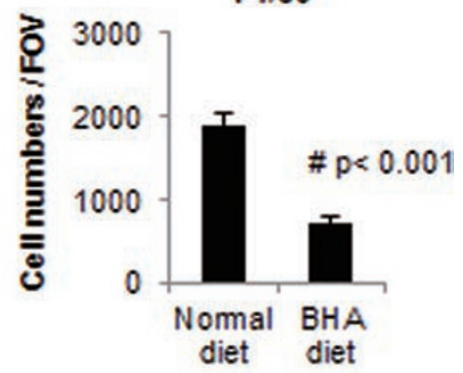

- Normal diet $(n=19)$

- BHA diet $(n=16)$
D

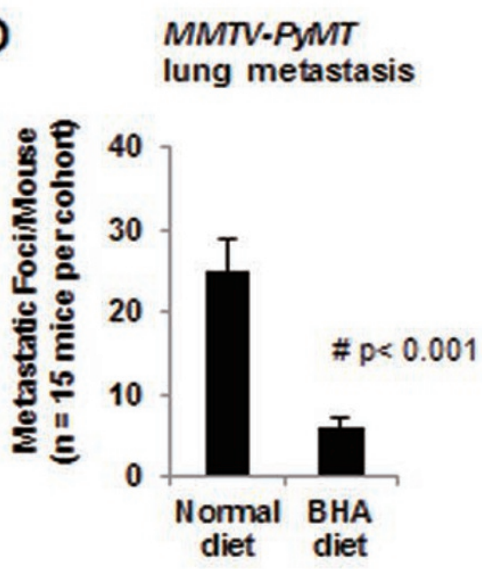

Figure 7 BHA blocks the occurrence of TAMs and suppresses primary tumor development and lung metastasis in MMTVPyMT breast cancer model. (A) MMTV-PyMT mice were maintained on normal or BHA diet at 3 weeks of age. Primary mammary tumors were collected at 100 days of age, sectioned and immunostained for F4/80. Representative image is shown in the left panel ( $T$, tumor area) and quantitative analysis is shown in the right panel. The F4/80 cells were evaluated by counting 10 high power fields $(20 \times)$ per lung section $(1 \mathrm{~mm}) /$ two tissue sections/mouse. $n=6$ mice. (B) Tumor burden of mice from (A) was determined by measuring primary tumors as described in the Materials and Methods. (C) Representative image of H\&E stained pulmonary adenoma metastasis of mice from A. (D) Metastaic lung tumor multiplicity of mice from A was determined by counting total tumor foci in five serial sections at $400 \mathrm{~mm}$ intervals. $n=15$; Error Bars: \pm SEM.

and an essential component of NOX, efficiently blocked M2 macrophage differentiation, suggesting that NOXmediated $\mathrm{O}^{2-}$ generation is required for M2 macrophage differentiation. However, as BHA, TEMPO, NAC and apocynin are general ROS inhibitors, other source of ROS, such as mitochondria-generated ROS, may also be involved in M2 differentiation. ROS is needed for certain downstream signaling pathways, such as the late-phase ERK activation. While all three MAPKs, ERK, JNK and p38, are activated at the early stage of monocytemacrophage differentiation, only the late ERK activation is affected by the inhibition of ROS. Our data indicated that this late ERK activation is essential for monocytemacrophage differentiation. Surprisingly, we found that 
A

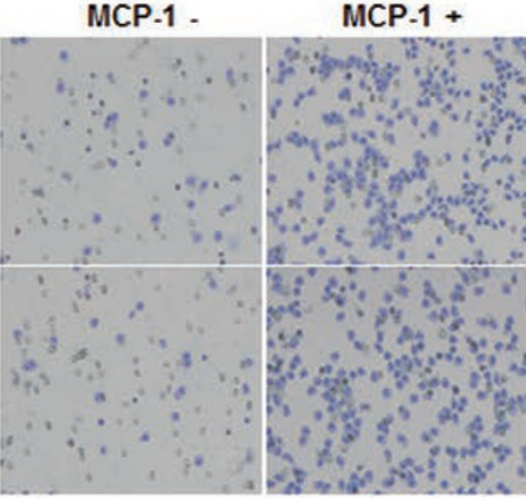

BHA -

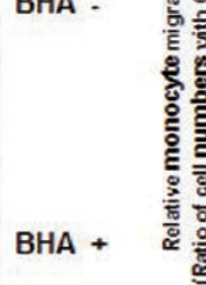

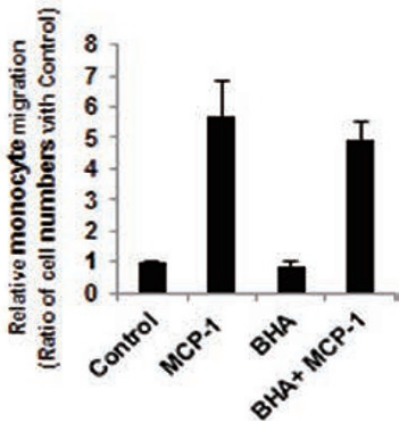

$\mathbf{B}$

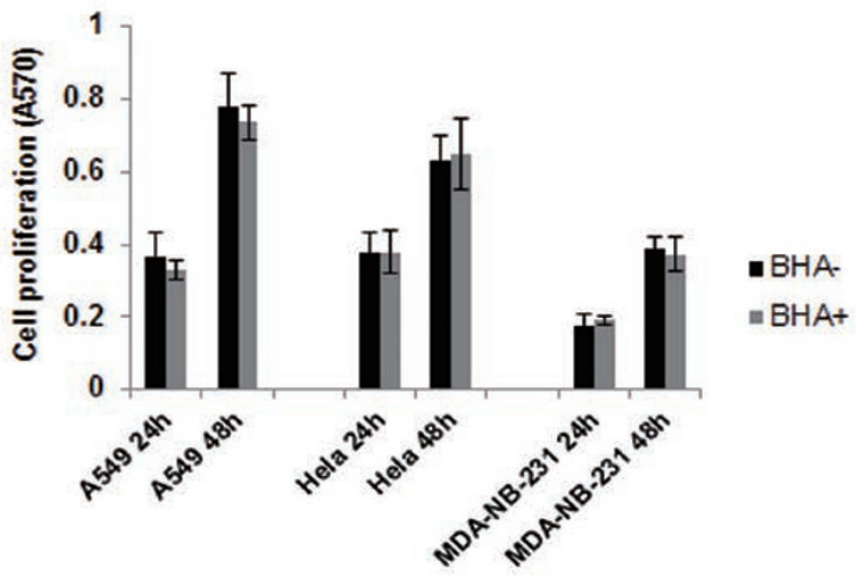

C

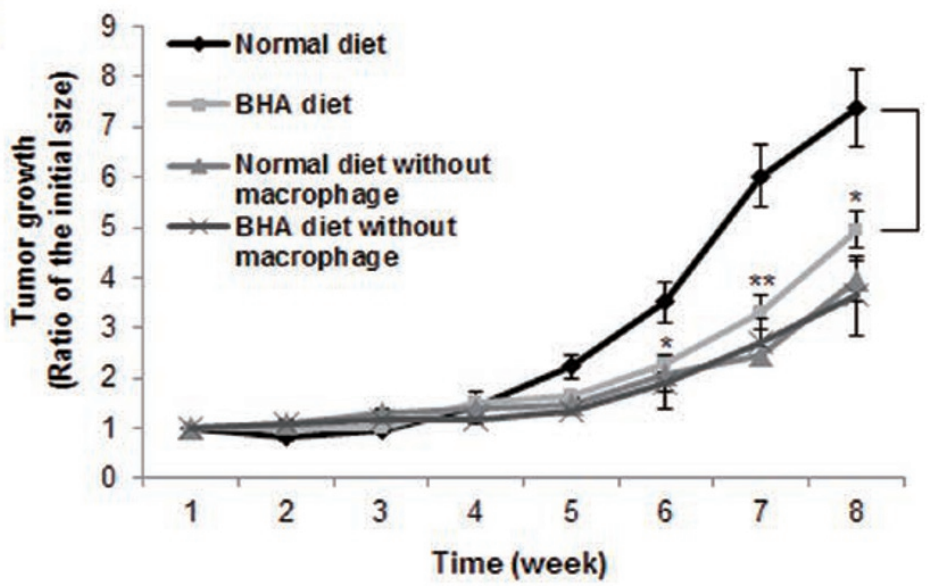

Figure 8 BHA has no effect on MCP-1-directed monocyte migration and tumor cell growth. (A) Elutriated human monocytes were treated with MCP-1 $(50 \mathrm{ng} / \mathrm{ml})$ and the cell migration was assayed by using Transwell inserts. Cells were pretreated with or without BHA $(100 \mu \mathrm{M})$ for $1 \mathrm{~h}$ prior to loading onto the migration chamber. Migrated cells were stained with crystal violet and evaluated by blind counting of the migrated cells on the lower surface of the membrane of 5 fields per chamber. Representative image is shown in the left panel and quantitative analysis is shown in the right panel. Data in monocyte migration detections represent the means \pm SD of nine determinants from 3 independently prepared human samples each with 3 measurements. (B) A549, Hela and MDA-NB-231 cells were treated with or without BHA and the cell proliferation were detected by the MTT assay at $24 \mathrm{~h}$ and $48 \mathrm{~h}$. The figure shows means \pm SD from three independent experiments. (C) Tumor growth curve of MDA-MB-231-tdTomato cells inoculated into mouse mammary fat pads is shown. Female athymic nu/nu mice with or without macrophage depletion were divided into normal $\mathrm{NIH}-31$ chow or $\mathrm{NIH}-31$ chow with $7.5 \mathrm{~g} / \mathrm{kg} \mathrm{BHA} 1$ week before the inoculation of tumor cells. Tumor size was measured for each animal every week starting from the day of the initial treatment. Relative tumor growth was normalized to week 1 . Results are given as means $\pm \operatorname{SEM}\left(n=6 /\right.$ group). ${ }^{*} P<0.05 ;{ }^{* \star} P<0.01$ compared with normal diet control group at matched time point. 
elimination of ROS only blocked the polarization of M-CSF-differentiated macrophages to M2, but not GMCSF-differentiated cells to M1 macrophages. When GM-CSF-differentiated cells are polarized to M1 macrophages, several signaling pathways including ERK are activated by LPS through TLR-4 [27]. The pathways directly activated by TLR-4, such as ERK activation, are not affected by the elimination of ROS. Therefore, when cells are polarized to M1 macrophages by LPS/INF $\gamma$, the inhibitory effect of BHA on GM-CSF-differentiated cells is overcome by TLR-4-mediated signaling. In contrast, during the polarization to M2 macrophages, IL-4 does not activate signaling pathways, such as ERK, which are blocked by BHA, in M-CSF-differentiated cells. Thus, elimination of ROS only affects the polarization to M2 but not M1 macrophages.

M-CSF-induced BMMs have been widely used to study mouse macrophages, however, slightly different from the human system, GM-CSF treatment of mouse monocytes results in a mixture of macrophages and DCs $[44,45]$. In M-CSF-induced BMMs, BHA blocked IL-4/ IL-10-triggered polarization to mouse M2 macrophages, but did not affect LPS/IFN $\gamma$-induced polarization to mouse M1 macrophages. Our data from studies on murine macrophage differentiation in vitro and in vivo suggest that the requirement of ROS in the differentiation of monocytes to M2 macrophages is a general phenomenon. Administering BHA in vivo could abrogate the differentiation of the low cytotoxic, growth-promoting M2 macrophages and have no effect on the differentiation of monocytes to the pro-inflammatory M1 macrophages.

TAMs produce a variety of factors to promote tumor growth and angiogenisis and to mediate immunosuppression [16]. Eliminating TAMs can effectively block tumorigenesis [20]. As TAMs are M2-like macrophages, we examined whether BHA can block the occurrence of TAMs in three different cancer models. We found that BHA indeed abolished the occurrence of TAMs in all three models regardless of when BHA was administered to mice during tumorigenesis. In the Urethane-induced lung cancer model, BHA eliminated the occurrence of TAMs with similar efficiency, no matter whether it was administered before or after Urethane treatment (Figure 5). In the $K-$ ras $^{L A 2}$ lung cancer model and MMTV-PyMT breast cancer model, administering BHA after tumor initiation $[40,46]$ also efficiently blocked the occurrence of TAMs (Figures 6 and 7). Consistent with previous reports that TAMs promote tumor initiation, progression and metastasis, our data indicated that BHA suppressed tumorigenesis in all three stages. We found that BHA blocks tumor initiation and progression in the Urethaneinduced lung cancer model, inhibits tumor progression in $K$-ras ${ }^{L A 2}$ lung cancer model, and reduces tumor progression and metastasis in MMTV-PyMT breast cancer model. While the effect of BHA on TAMs is similar in these three models, BHA inhibits tumorigenesis most effectively when it was administered before Urethane treatment, supporting the notion that TAMs play a critical role in tumor initiation. When BHA is administered after tumor initiation, it markedly suppresses tumor growth and reduced metastasis (Figures 6 and 7). Importantly, our data indicated that the differentiation of inflammatory $\mathrm{M} 1$ macrophages or $\mathrm{F} 4 / 80^{+}$macrophages in noncancer mice is not affected by BHA (Figures 4D, 5A and unpublished data). Considering the importance of TAMs in tumorigenesis, our study clearly demonstrated that inhibiting the occurrence of TAMs by BHA contributes to its inhibition of tumorigenesis.

The antitumor activity of BHA has been reported in several carcinogen-induced animal tumor models [37, 47]. However, as BHA was administered only temporarily or periodically, the inhibitory effect of BHA on tumorigenesis is relatively small in these studies. Our finding that BHA blocks the ROS-mediated differentiation of M2 macrophages demonstrated the importance of continuous administration of BHA to block the occurrence of TAMs and efficiently suppress tumorigenesis. More importantly, we found that BHA has no effect on the proliferation of a variety of tumor cells in vitro. In the breast cancer xenograft model, mice fed with BHA food suppressed tumor growth compared with mice fed with normal food. As TAMs also play a pivotal role in tumor growth of xenograft models and Clodronate liposomemediated TAM depletion inhibits tumor growth in athy$\mathrm{mic} \mathrm{nu} / \mathrm{nu}$ mice [48], the effect of BHA in this model could be the result of the inhibition effect of BHA on TAMs. When macrophages were depleted in this model, BHA did not inhibit tumor growth, confirming that BHA suppressed tumorigenesis by targeting TAMs but not by directly affecting tumor cells. Additionally, BHA had no effect on MCP-1-induced monocyte migration, suggesting that BHA did not inhibit monocyte recruitment to the tumor tissue. Although BHA could have multiple targets during tumor development, our study suggests the possibility of BHA as an inhibitor of cancer development achieved mainly by blocking M2 macrophages, rather than by affecting other factors, such as tumor cell growth or TAM recruitment. As a cancer prevention and therapeutic agent, BHA may be more potent if it inhibits multiple components of tumorigenesis. Our data clearly demonstrated that the continuous administration of ROS inhibitors for targeting TAMs may be a potentially effective method for cancer treatment. As the ultimate goal of biomedical research is to translate the findings of basic 
research into new treatments of diseases, our current work is one of the examples that have the potential to translate the basic research into immediate applications to greatly improve human health.

\section{Materials and Methods}

\author{
Animals \\ $K$-ras ${ }^{L A 2}$ mice were from NCI mouse repository (NCI-Freder- \\ ick). $M M T V-P y M T$ mice, $\mathrm{A} / \mathrm{J}$ mice and $\mathrm{C} 57 \mathrm{BL} / 6$ were from the \\ Jackson Laboratory. Mice were maintained under pathogen-free \\ conditions, and experimental protocols were approved by NCI, \\ following NIH guidelines.
}

\section{Reagents and antibody}

BHA, Apocynin, NAC, LPS and Urethane were obtained from Sigma; anti-phospho-ERK and anti-ERK from Cell signaling; Anti-phospho JNK and Lucigenin from Invitrogen; anti-Ym-1 from STEMCELL Technologies; anti-Arginase I from Santa Cruz; antiiNOS, anti-JNK, CD11b-PE, CD86-PE, CD163-PE, anti-Ly6C from BD Biosciences; anti-F4/80, F4/80-APC, anti-IL-4 from eBiosciences; anti-RELM $\alpha$ from Peprotech; SP600125, U0126, and SB203580 were from Calbiochem; Recombinant human GMCSF, M-CSF, IL-4, IFN $\gamma$, and recombinant mouse M-CSF, IL-4, IFN $\gamma$ from R\&D Systems.

\section{Cell culture}

Elutriated human monocytes from blood were obtained from NIH Blood bank (according to NIH protocol 99CC0168). Monocytes were cultured in RPMI-1640 medium supplemented with $10 \%$ (vol/vol) FBS and $2 \mathrm{mM}$ glutamine, with penicillin $(100 \mathrm{U} /$ $\mathrm{ml})$ and streptomycin $(100 \mu \mathrm{g} / \mathrm{ml})$. For differentiation, cells were cultured for 6 days in the presence of recombinant human GMCSF $(10 \mathrm{ng} / \mathrm{ml})$ or M-CSF $(25 \mathrm{ng} / \mathrm{ml})$. For M1 polarization, monocytes treated with GM-CSF for 6 days were then treated with LPS $(100 \mathrm{ng} / \mathrm{ml})$ and IFN $\gamma(20 \mathrm{ng} / \mathrm{ml})$. For M2 polarization, monocytes treated with M-CSF for 6 days were then treated with IL-4 (25 ng/ $\mathrm{ml})$.

\section{$\mathrm{O}^{2-}$ generation assays}

$\mathrm{O}^{2-}$ generation was measured by $\mathrm{O}^{2-}$-dependent lucigenin chemiluminescence. Approximately $1 \times 10^{5}$ cells in $1 \mathrm{ml}$ HBSS were incubated with Lucigenin $(200 \mathrm{mM})$ for $5 \mathrm{~min}$. Chemiluminescence was measured at $37^{\circ} \mathrm{C}$.

\section{M1/M2 macrophage differentiation in vivo}

To generate M1 macrophages in vivo, 8-week-old female A/ $\mathrm{J}$ mice were maintained on normal or BHA diet for 2 weeks and thioglycolate-activated M1 macrophages were isolated by peritoneal lavage with $10 \mathrm{ml}$ of ice-cold PBS for 4 days after intraperitoneal injection of $1 \mathrm{ml} \mathrm{3 \%}$ sterile thioglycolate. The cells from thioglycolate-induced peritonitis were co-stained with anit-Ly6C and anti-F4/80 antibodies and analyzed by flow cytometry.

To generate M2 macrophages in vivo, we injected (i.p.) 8-weekold female $\mathrm{A} / \mathrm{J}$ mice that were on normal or BHA diet for 2 weeks with $200 \mu \mathrm{l}$ of a IL-4c (combination of a 2:1 ratio of recombinant mouse IL-4 (rmIL-4; $5 \mu \mathrm{g}$ ) and anti-IL-4 Ab (clone 11B11; $25 \mu \mathrm{g}$ in PBS)), or PBS vehicle control on days 0 and 2 . On day 4 , the cells from IL-4c-induced peritonitis were isolated by peritoneal lavage with $10 \mathrm{ml}$ of ice-cold PBS and co-stained with anti-F4/80 and anti-RELM $\alpha$ antibodies and analyzed by FACS.

\section{Tumor models}

Urethane model: 8-week-old female A/J mice that were weight and age matched were used for experiments. Tumors were induced by single i.p. injection of $1 \mathrm{~g} / \mathrm{kg}$ Urethane. Animals were then divided into normal NIH-31 chow or NIH-31 chow with $7.5 \mathrm{~g} / \mathrm{kg}$ BHA. After 6 months, lungs were excised and evaluated for tumors.

Kras model: 3-week-old male $K$-ras ${ }^{L A 2}$ mice were divided into normal NIH-31 chow or NIH-31 chow with $7.5 \mathrm{~g} / \mathrm{kg}$ BHA. At 5 months of age, lungs were excised and evaluated for tumors.

Breast tumor model: 3-week-old female $M M T V-P y M T$ mice were divided into normal NIH-31 chow or NIH-31 chow with 7.5 $\mathrm{g} / \mathrm{kg}$ BHA. Breast and lungs from 100-day-old mice were excised and evaluated for tumors.

\section{Monocyte migration assays}

Human monocytes were measured in a Boyden chamber migration assay using Transwell inserts with an $8-\mu \mathrm{m}$ porous membrane (BD Biosciences). Freshly elutriated human monocytes were suspended at $2 \times 10^{6}$ cells $/ \mathrm{ml}$ in RPMI 1640 containing $0.1 \mathrm{mg} / \mathrm{ml}$ BSA. A $0.25-\mathrm{ml}$ cell suspension (final concentration, $5 \times 10^{5}$ cells/ well) was added to the upper compartment, and cells were incubated at $37{ }^{\circ} \mathrm{C}\left(95 \%\right.$ air- $\left.5 \% \mathrm{CO}_{2}\right)$. Medium containing $50 \mathrm{ng} / \mathrm{ml} \mathrm{MCP-}$ 1 was placed in the lower chamber. After allowing cell migration for $30 \mathrm{~min}$, cells were removed from the upper side of membranes with a cotton swab, and the migrated cells were fixed with paraformaldehyde and stained with crystal violet. Cell migration was quantified by blind counting of the migrated cells on the lower surface of the membrane of five fields per chamber under microscope. To evaluate the effect of BHA on monocyte migration, cells were pretreated with BHA $(100 \mu \mathrm{M})$ for $1 \mathrm{~h}$ prior to loading onto the migration chamber.

\section{Cell proliferation assay}

A549, Hela and MDA-NB-231 cells were seeded in 12-well plates at 50000 cells per well and cultured overnight. Cells were treated with or without BHA $(100 \mu \mathrm{M})$. The MTT (3-(4,5-dimethylthiazol-2-yl)-2,5-diphenyltetrazoliumbromide) assay was used to determine relative cell viability at $24 \mathrm{~h}$ and $48 \mathrm{~h}$ after BHA treatment. Fifty microlitres of MTT solution was added to $100 \mathrm{ml}$ of culture media and incubated for $4 \mathrm{~h}$ at $37{ }^{\circ} \mathrm{C}$, and the optical density was measured at $570 \mathrm{~nm}$. Each experiment was performed in triplicates and repeated three times.

\section{tdTomato-expressing breast cancer cell line and breast can- cer xenograft model}

Female athymic nu/nu mice (6-week-old; The Jackson Laboratory) were maintained in microisolation cages under specific pathogen-free conditions. MDA-MB-231-tdTomato cells (gift from Dr Kathleen Kelly, NCI) were resuspended in PBS and Matrigel (1:1 v/v). Two million MDAMB-231-tdTomato cells were orthotopically inoculated in the mammary fat pad of anesthetized female athymic nude mice. Animals were divided into groups maintained on normal NIH-31 chow or NIH-31 chow with $7.5 \mathrm{~g} /$ $\mathrm{kg}$ BHA starting 1 week before the inoculation of tumor cells. For 
macrophage depletion, the mice with/without BHA chow received Clophosome-A-Clodronate Liposomes (Anionic) or control liposome (FormuMax) by intraperitoneal (i.p.) injection as initial dose of $0.2 \mathrm{ml}$ per mouse, followed by $0.1 \mathrm{ml}$ per mouse once a week for 8 weeks. Tumor xenografts reached their final experimental size within 6-8 weeks. In vivo whole-body fluorescence imaging of MDA-MB-231-tdTomato tumor xenografts was performed using a commercially available optical animal imaging system, In-vivo Xtreme (Bruker) under isofluorane anesthesia at different times. Relative tumor growth was normalized to week one.

\section{Statistical analysis}

Statistical analyses were performed using GraphPad Prism and/ or Aperio ImageScope Software. Two group comparisons were performed using Student's $t$-test. All $P$-values less than 0.05 were considered statistically significant.

Detailed methods are described in the Supplementary information, Data S1.

\section{Acknowledgments}

We thank AA Molinolo (NIH/NIDCR) for his expertise in histopathology and the National Institutes of Health Blood Bank for elutriated monocytes. We also thank Dr JJ Yin (NCI/CCBB) for helping set up the breast cancer xenograft model, V Diaz (NIH/ NINDS) for the mouse imaging and B Barreff (NCI/CCBB) for helping with analysis of mouse image data. This study was supported by the Intramural Research Program of The Center for Cancer Research, NCI, NIH.

\section{References}

1 Martinez FO, Helming L, Gordon S. Alternative activation of macrophages: an immunologic functional perspective. Annu Rev Immunol 2009; 27:451-483.

2 Fairweather D, Cihakova D. Alternatively activated macrophages in infection and autoimmunity. J Autoimmun 2009; 33:222-230.

3 Mantovani A, Allavena P, Sica A, Balkwill F. Cancer-related inflammation. Nature 2008; 454:436-444.

4 Gordon S, Taylor PR. Monocyte and macrophage heterogeneity. Nat Rev Immunol 2005; 5:953-964.

5 Bourette RP, Rohrschneider LR. Early events in M-CSF receptor signaling. Growth Factors 2000; 17:155-166.

6 Martinez-Moczygemba M, Huston DP. Biology of common beta receptor-signaling cytokines: IL-3, IL-5, and GM-CSF. $J$ Allergy Clin Immunol 2003; 112:653-665; quiz 666.

7 Wang X, Studzinski GP. Activation of extracellular signalregulated kinases (ERKs) defines the first phase of 1,25-dihydroxyvitamin D3-induced differentiation of HL60 cells. J Cell Biochem 2001; 80:471-482.

8 Himes SR, Sester DP, Ravasi T, Cronau SL, Sasmono T, Hume DA. The JNK are important for development and survival of macrophages. J Immunol 2006; 176:2219-2228.

9 Wang Y, Zeigler MM, Lam GK, et al. The role of the NADPH oxidase complex, p38 MAPK, and Akt in regulating human monocyte-macrophage survival. Am J Respir Cell Mol Biol 2007; 36:68-77.
10 Suzu S, Hiyoshi M, Yoshidomi Y, et al. M-CSF-mediated macrophage differentiation but not proliferation is correlated with increased and prolonged ERK activation. J Cell Physiol 2007; 212:519-525.

11 Zhang Y, Morgan MJ, Chen K, Choksi S, Liu ZG. Induction of autophagy is essential for monocyte-macrophage differentiation. Blood 2012; 119:2895-2905.

12 Gordon S, Martinez FO. Alternative activation of macrophages: mechanism and functions. Immunity 2010; 32:593-604.

13 Verreck FA, de Boer T, Langenberg DM, van der Zanden L, Ottenhoff TH. Phenotypic and functional profiling of human proinflammatory type- 1 and anti-inflammatory type- 2 macrophages in response to microbial antigens and IFN-gammaand CD40L-mediated costimulation. J Leukoc Biol 2006; 79:285-293.

14 Mantovani A, Sozzani S, Locati M, Allavena P, Sica A. Macrophage polarization: tumor-associated macrophages as a paradigm for polarized M2 mononuclear phagocytes. Trends Immunol 2002; 23:549-555.

15 Condeelis J, Pollard JW. Macrophages: obligate partners for tumor cell migration, invasion, and metastasis. Cell 2006; 124:263-266.

16 Qian BZ, Pollard JW. Macrophage diversity enhances tumor progression and metastasis. Cell 2010; 141:39-51.

17 Mehrpour M, Esclatine A, Beau I, Codogno P. Overview of macroautophagy regulation in mammalian cells. Cell Res 2010; 20:748-762.

18 Mantovani A, Sica A. Macrophages, innate immunity and cancer: balance, tolerance, and diversity. Curr Opin Immunol 2010; 22:231-237.

19 Pollard JW. Trophic macrophages in development and disease. Nat Rev Immunol 2009; 9:259-270.

20 Baay M, Brouwer A, Pauwels P, Peeters M, Lardon F. Tumor cells and tumor-associated macrophages: secreted proteins as potential targets for therapy. Clin Dev Immunol 2011; 2011:565187.

21 Ruffell B, Affara NI, Coussens LM. Differential macrophage programming in the tumor microenvironment. Trends Immunol 2012; 33:119-126.

22 Finkel T, Holbrook NJ. Oxidants, oxidative stress and the biology of ageing. Nature 2000; 408:239-247.

23 Tatla S, Woodhead V, Foreman JC, Chain BM. The role of reactive oxygen species in triggering proliferation and IL-2 secretion in T cells. Free Radic Biol Med 1999; 26 :14-24.

24 Goldstone SD, Milligan AD, Hunt NH. Oxidative signalling and gene expression during lymphocyte activation. Biochim Biophys Acta 1996; 1314:175-182.

25 Sardina JL, López-Ruano G, Sánchez-Abarca LI, Pérez-Simón JA, Gaztelumendi A, Trigueros C, et al. p22phox-dependent NADPH oxidase activity is required for megakaryocytic differentiation. Cell Death Differ 2010; 17:1842-1854.

26 Kim YS, Morgan MJ, Choksi S, Liu ZG. TNF-induced activation of the Nox1 NADPH oxidase and its role in the induction of necrotic cell death. Mol Cell 2007; 26:675-687.

27 Biswas SK, Mantovani A. Macrophage plasticity and interaction with lymphocyte subsets: cancer as a paradigm. Nat Immunol 2010; 11:889-896.

28 Martinez FO, Sica A, Mantovani A, Locati M. Macrophage activation and polarization. Front Biosci 2008; 13:453-461. 
29 Kim KS, Choi HW, Yoon HE, Kim IY. Reactive oxygen species generated by NADPH oxidase 2 and 4 are required for chondrogenic differentiation. J Biol Chem 2010; 285:4029440302.

30 Miyano K, and Sumimoto H. Assessment of the role for Rho family GTPases in NADPH oxidase activation. Methods Mol Biol 2012; 827:195-212.

31 Biswas SK, Mantovani A. Orchestration of metabolism by macrophages. Cell Metab 2012; 15:432-437.

32 Munder M, Eichmann K, Morán JM, Centeno F, Soler G, Modolell M. Th1/Th2-regulated expression of arginase isoforms in murine macrophages and dendritic cells. J Immunol 1999; 163:3771-3777.

33 Sica A, Mantovani A. Macrophage plasticity and polarization: in vivo veritas. $J$ Clin Invest $2012 ; \mathbf{1 2 2}: 787-795$.

34 Khazen W, M'bika JP, Tomkiewicz C, et al. Expression of macrophage-selective markers in human and rodent adipocytes. FEBS Lett 2005; 579:5631-5634.

35 Jenkins SJ, Ruckerl D, Cook PC, et al. Local macrophage proliferation, rather than recruitment from the blood, is a signature of TH2 inflammation. Science 2011; 332:1284-1288.

36 Witschi HP. Enhancement of tumor formation in mouse lung by dietary butylated hydroxytoluene. Toxicology 1981; 21:95104.

37 Redente EF, Orlicky DJ, Bouchard RJ, Malkinson AM. Tumor signaling to the bone marrow changes the phenotype of monocytes and pulmonary macrophages during Urethane-induced primary lung tumorigenesis in A/J mice. Am J Pathol 2007; 170:693-708.

38 Levine B, Klionsky DJ. Development by self-digestion: molecular mechanisms and biological functions of autophagy. Dev Cell 2004; 6:463-477.

39 Takahashi H, Ogata H, Nishigaki R, Broide DH, Karin M. Tobacco smoke promotes lung tumorigenesis by triggering IKKbeta- and JNK1-dependent inflammation. Cancer Cell 2010; 17:89-97.

40 Johnson L, Mercer K, Greenbaum D, et al. Somatic activation of the K-ras oncogene causes early onset lung cancer in mice. Nature 2001; 410:1111-1116.
41 Vellai T, Takacs-Vellai K, Zhang Y, Kovacs AL, Orosz L, Müller F. Genetics: influence of TOR kinase on lifespan in $C$. elegans. Nature 2003; 426:620.

42 Lin EY, Nguyen AV, Russell RG, Pollard JW. Colony-stimulating factor 1 promotes progression of mammary tumors to malignancy. $J$ Exp Med 2001; 193:727-740.

43 DeNardo DG, Barreto JB, Andreu P, et al. CD4(+) T cells regulate pulmonary metastasis of mammary carcinomas by enhancing protumor properties of macrophages. Cancer Cell 2009; 16:91-102.

44 Inaba K, Steinman RM, Pack MW, et al. Identification of proliferating dendritic cell precursors in mouse blood. J Exp Med 1992; 175:1157-1167.

45 Fleetwood AJ, Lawrence T, Hamilton JA, Cook AD. Granulocyte-macrophage colony-stimulating factor (CSF) and macrophage CSF-dependent macrophage phenotypes display differences in cytokine profiles and transcription factor activities: implications for CSF blockade in inflammation. J Immunol 2007; 178:5245-5252.

46 Kuma A, Hatano M, Matsui M, et al. The role of autophagy during the early neonatal starvation period. Nature 2004; 432:1032-1036.

47 Wattenberg LW. Protective effects of 2(3)-tert-butyl-4-hydroxyanisole on chemical carcinogenesis. Food Chem Toxicol 1986; 24:1099-1102.

48 Zeisberger SM, Odermatt B, Marty C, Zehnder-Fjällman AH, Ballmer-Hofer K, Schwendener RA. Clodronate-liposomemediated depletion of tumour-associated macrophages: a new and highly effective antiangiogenic therapy approach. $\mathrm{Br} \mathrm{J}$ Cancer 2006; 95:272-281.

(Supplementary information is linked to the online version of the paper on the Cell Research website.)

(c) (1) () $(-)$ This work is licensed under the Creative Commons Attribution-NonCommercial-No Derivative Works 3.0 Unported License. To view a copy of this license, visit http:// creativecommons.org/licenses/by-nc-nd/3.0 